\title{
Expected performances of the PROBA-3/ASPIICS solar coronagraph: Simulated data
}

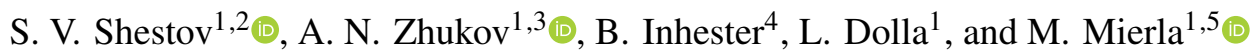 \\ ${ }^{1}$ Solar-Terrestrial Centre of Excellence - SIDC, Royal Observatory of Belgium, Avenue Circulaire 3, 1180 Brussels, Belgium \\ e-mail: s.shestov@oma.be \\ 2 Lebedev Physical Institute, Leninskii prospekt, 53, 119991 Moscow, Russia \\ 3 Skobeltsyn Institute of Nuclear Physics, Moscow State University, Leninskie gory, 119991 Moscow, Russia \\ 4 Max Planck Institut für Sonnensystemforschung, Justus-von-Liebig-Weg 3, 37077 Göttingen, Germany \\ 5 Institute of Geodynamics of the Romanian Academy, 19-21 Jean-Louis Calderon St., 020032 Bucharest, Romania
}

Received 1 February 2021 / Accepted 19 April 2021

\begin{abstract}
Context. The Association of Spacecraft for Polarimetric and Imaging Investigation of the Corona of the Sun (ASPIICS) is a novel externally occulted solar coronagraph that will be launched on board the Project for On-Board Autonomy (PROBA-3) mission in 2023. The external occulter will be placed on the first satellite $\sim 150 \mathrm{~m}$ ahead of the second satellite, which will carry an optical instrument. During 6 hours per orbit, the satellites will fly in a precise formation and will constitute a giant externally occulted coronagraph. The large distance between the external occulter and the primary objective will allow observations of the white-light solar corona starting from extremely low heights of $\sim 1.1 R_{\odot}$.

Aims. Developing and testing of algorithms for the scientific image processing requires understanding of all the optics-related and detector-related effects of the coronagraph, development of appropriate physical and numerical models, and preparation of simulated images that include all these effects. At the same time, an analysis of the simulated data gives valuable information about the performance of the instrument, the suitable observation regime, and the amount of telemetry.

Methods. We used available physical models of the instrument and implemented them as a software to generate simulated data. We analyzed intermediate and complete simulated images to obtain a better understanding of the performance of ASPIICS, in particular, to predict its photometric sensitivity, effect of noise, suitable exposure times, etc.

Results. The proposed models and algorithms are used not only to create the simulated data, but also to form the basis for the scientific processing algorithms to be applied during on-ground ASPIICS data processing. We discuss the possible effect of noise and the uncertainty of the calibration factors on the accuracy of final data, and propose suitable exposure times.
\end{abstract}

Key words. Sun: corona - instrumentation: high angular resolution - telescopes - methods: numerical

\section{Introduction}

The solar corona is the outer layer of the solar atmosphere (Golub \& Pasachoff 1997). It consists of highly ionized plasma that is structured by the magnetic field. In the solar corona a number of important but still not well-understood phenomena take place, such as the initiation and acceleration of coronal mass ejections (CMEs), and the acceleration of the solar wind. The solar corona is routinely observed from space today by extreme ultraviolet (EUV) telescopes and white-light coronagraphs. In the EUV spectral range, the emission decays in general, depending on the excitation mechanism, more rapidly with height than Thomson-scattered white-light emission. Thus EUV telescopes usually observe corona up to a relatively moderate heights of about $2 R_{\odot}$ (throughout the manuscript, we denote the solar angular radius by $R_{\odot}$ ). In contrast, white-light coronagraphs just start to observe from these heights: The signal is proportional to the electron density $n_{e}$ and decays much more slowly with height, but the diffraction from the solar disk precludes a reduction of the minimum observational height (Koutchmy 1988; Bout et al. 2000).

The Association of Spacecraft for Polarimetric and Imaging Investigation of the Corona of the Sun (ASPIICS) coronagraph is a novel white-light solar coronagraph that will perform regular observations of the corona within the field of view (FOV) from $\sim 1.1 R_{\odot}$ up to $3.0 R_{\odot}$ (Lamy et al. 2010; Renotte et al. 2015; Galano et al. 2018). This will be possible owing to the European Space Agency formation-flying mission PROBA-3 (Tiraplegui et al. 2019), the third mission of the Project for OnBoard Autonomy (PROBA). In PROBA-3/ASPIICS the external occulter (EO) will be placed on the first satellite, and the telescope will be placed on the second. During the $\sim 6$ hours out of 19.6-hour orbit, the satellites will fly in precise formation, placing the telescope in the shadow of the EO with an accuracy of about a few millimeters (Tiraplegui et al. 2019; Galano et al. 2019). The telescope, which is developed by Centre Spatial de Liège, will be equipped with a $2048 \times 2048$ pixel complementary metal oxide semiconductor (CMOS) camera with a pixel angular scale $\sim 2.81$ arcsec, and a filter wheel with six positions to choose between three spectral filters and three linear polarizers oriented at $60^{\circ}$. The huge inter-satellite distance (ISD) with a nominal value of $144348 \mathrm{~mm}$ will allow unvignetted observations of the solar corona starting already from $\approx 1.17 R_{\odot}$.

The low minimum height of the observed corona together with a small vignetted zone in the images of ASPIICS are in stark contrast to other space solar coronagraphs, 
from the Large Angle Spectroscopic Coronagraph (LASCO) C2 (Brueckner et al. 1995; Llebaria et al. 2004) on board the Solar and Heliospheric Observatory (SOHO), to Metis (Antonucci et al. 2020) on board Solar Orbiter. The proximity of the EO to the primary objective, dictated by a single-instrument design, results in the vignetting of the whole FOV of all the previous instruments. Vignetting not only reduces the photometric sensitivity, but also results in the degradation of the spatial resolution of the instrument due to diffraction at smaller apertures (Koutchmy 1988). In ASPIICS, the vignetted zone is significantly smaller, and in addition, the absence of pilons supporting the occulter causes almost the whole FOV to be unvignetted. Thus, from the point of view of coronal observations, ASPIICS forms quite a simple camera that can be characterized by a limited number of parameters, such as focal length, flat field, and photometric sensitivity.

The relatively small overoccultation by the EO (its angular size is just $1.057 R_{\odot}$ ) will result in an essential amount of diffracted light entering the optical system. In the focal plane it will have the shape of a bright diffracted fringe with an intensity comparable to that of the corona, and with exponentially decaying wings. The intensity of the diffracted light depends not only on the internal diaphragms of the telescope, such as the internal occulter and the Lyot stop (Rougeot et al. 2017, hereafter RR17), but also on the angular size of the Sun, and on the mutual in-orbit coalignment of the telescope, the EO, and the Sun (Shestov \& Zhukov 2018, hereafter SZ18).

There will be other optical effects such as scattered light and ghost light that will be present in the registered images. The CMOS-based detector of ASPIICS will have all the typical effects such as dark current, bias, response nonlinearity, hot pixels, and readout noise, which will be present in the images and ultimately complicate processing and analyzing them. In addition, in order to catch the high dynamic range of the coronal brightness, ASPIICS will work in a multi-exposure regime: during nominal operations, the images will be registered in sequences with different exposure times, provisionally $0.1 \mathrm{~s}, 1.0$, and $10.0 \mathrm{~s}$.

The on-ground processing of the ASPIICS data (Shestov et al. 2020) will remove all the known opticsand detector-related effects from the images and apply radiometric calibrations. It will finally assemble full-dynamic-range images or additionally apply some processing to polarized images to retrieve the polarization parameters. The processing extensively relies on physical models, therefore we also call it scientific processing.

The performance of the coronagraph, which includes its ability to detect faint or low-contrast structures in the corona in the presence of stray light and noises, the precision of the final data, as well as the amount of information transmitted to the ground, will significantly depend on the actual set of parameters: brightness of the coronal scene, working spectral passband, exposure time, performance of the detector, mutual coalignment in space, etc. Therefore a proper modeling of the images, or end-to-end image simulation, is essential for the project. End-to-end science data simulation has been used with great success for earlier space missions such as Kepler (Bryson et al. 2010), the Transiting Exoplanet Survey Satellite (TESS, Jenkins et al. 2018), and the Characterising Exoplanet Satellite (CHEOPS, Futyan et al. 2020), but to our knowledge, it has not been used in solar whitelight coronagraphy. Creating simulated data, that is, artificial images resembling those to be actually registered by the coronagraph, may serve two purposes: First, it will provide the data that can be used as input to facilitate the development and testing of the on-ground processing algorithms, and second, it will to provide a more detailed understanding of the coronagraph characteristics and performance. The second purpose also supports design decisions to be taken during the development of the instrument, and will support commanding of the instrument during in-flight operations.

The aim of the present paper is to discuss available physical models and show the approach for the generation of the simulated data. We analyze the influence of the effects on the images registered by the telescope. We also discuss the effect of random noise and systematic errors and discuss the provisional accuracy of the processing. The paper is structured as follows: in Sect. 2 we recall the optical layout of ASPIICS, in Sect. 3 we depict the general framework for the simulated image generation. In Sect. 4 we describe the input coronal images, in Sects. 5 and 6 we discuss optics- and detector-related effects in detail, and in Sect. 7 we finally introduce noise to complete the simulated data generation. In Sect. 8 we analyze the obtained results: We present complete simulated images (Sect. 8.1), analyze the effects of noise (Sect. 8.2) and systematic errors (Sect. 8.3), and the visibility of stars (Sect. 8.4). In Sect. 9 we discuss the limitations of the current physical models, and we conclude in Sect. 10.

\section{Optical layout}

A detailed description of the optical layout of ASPIICS is given in Galy et al. (2015), and a functional description is presented in RR17 and SZ18. The optical layout, however, is essential for understanding many of the effects that are to be modeled, so we recall it here.

The optical layout is given in Fig. 1. The external occulter (not shown in the figure) with a diameter of $1.42 \mathrm{~m}$ is situated $z_{0} \sim 144.3 \mathrm{~m}$ in front of the entrance aperture. The entrance aperture with a diameter of $\varnothing=50 \mathrm{~mm}$ is situated in front of the primary objective (PO). The PO, a cemented doublet with the focal length $f=330.35 \mathrm{~mm}$, creates the image of the corona in the primary focus. The light, diffracted on the EO, will be focused slightly behind $(\Delta=0.76 \mathrm{~mm})$ the primary focus, in a plane conjugated to the EO plane. The internal occulter (IO) situated in this plane is oversized with respect to the bright diffracted fringe of the EO. The IO is an opaque coating deposited on the flat surface of the field lens O2. The diffracted bright fringe of the aperture will be cut off by the Lyot stop. The relay lens $\mathrm{O} 3$, consisting of four optical elements, projects the primary focus onto the detector plane, providing an effective focal length $f_{\text {eff }}=734.6 \mathrm{~mm}$. The filter wheel contains three interference filters: One wide-band filter (WBF) for the range 535-565 nm, two narrow-band filters $(\mathrm{NBF})$ with a full width at half maximum (FWHM) of $\Delta \lambda \sim 0.6 \mathrm{~nm}$ centered at $530.45 \mathrm{~nm}$ (Fe XIV line) and $F W H M \sim 2 \mathrm{~nm}$ centered at $587.7 \mathrm{~nm}$ (D3 line of He I), and three polarizers rotated one to another at $60^{\circ}$ and coupled with the WBF filters. The filters are placed $\sim 40 \mathrm{~mm}$ in front of the detector. A so-called detector protective glass containing $50 \%$ neutral density (ND50\%) filter is situated just $\sim 5 \mathrm{~mm}$ in front of the detector surface.

The telescope is equipped with a $2048 \times 2048$ pixel CMOS detector with a pixel size $\mathrm{d} x=10 \mu \mathrm{m}$, providing a pixel plate scale of 2.81 arcsec. The outer FOV of the coronagraph is determined by the detector size, and the inner FOV is determined by the size of the internal occulter, which allows coverage from $\sim 1.1 R_{\odot}$ to $3.0 R_{\odot}$. In addition to the extremely low minimum FOV and narrow vignetted zone, the unique optical configuration enables a very good optimization of the optical layout: the 


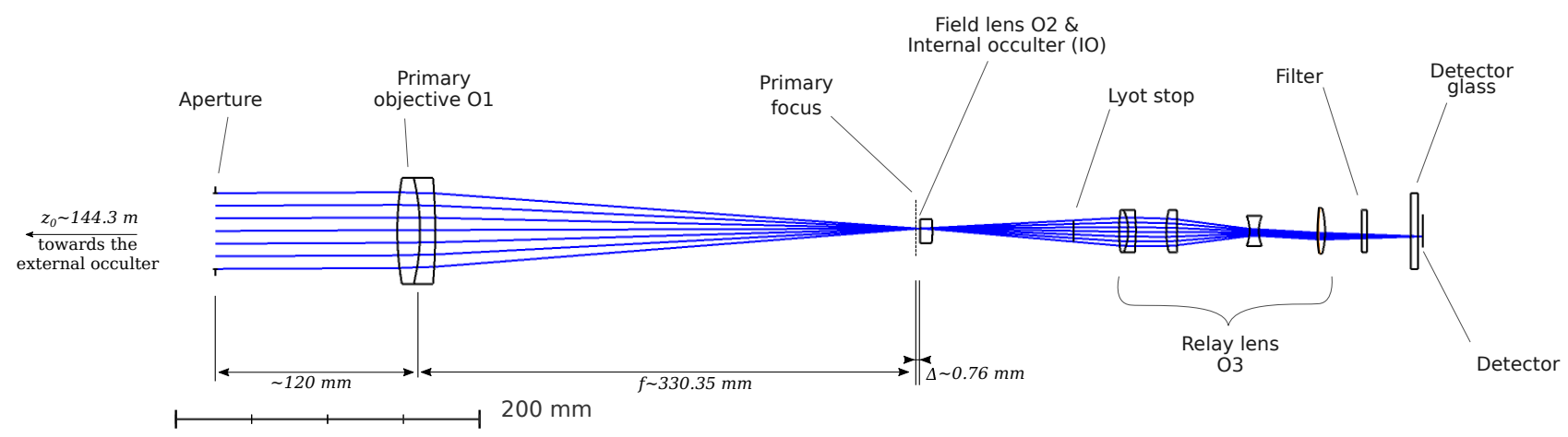

Fig. 1. Optical layout of the ASPIICS coronagraph. The external occulter (not shown) is situated $z_{0} \approx 144.3 \mathrm{~m}$ to the left. The entrance aperture is situated $120 \mathrm{~mm}$ in front of the primary objective with $f=330.35 \mathrm{~mm}$. The field lens and the internal occulter are situated $\sim 331.15 \mathrm{~mm}$ to the right of the PO. The Lyot stop is situated in a conjugate plane to the entrance aperture, before the relay lens O3. The relay lens projects the image from the primary focus onto the detector plane. The filter is situated between the last element of $\mathrm{O} 3$ and the detector. The detector is equipped by a protective glass with a $50 \%$ neutral density filter. The image and the elements are to scale.

expected point spread function (PSF) of the telescope is diffraction limited and is slightly larger than 1 pixel (Galy et al. 2015).

\section{General model}

We start by considering a coronal scene with known brightness that is being imaged by a perfect telescope, with an ideal 1-pixelsize PSF, and all known other characteristics such as aperture size, focal length, detector sensitivity, and filter passbands. We assume the telescope has no optical ghosts and no scattering. In the next few paragraphs we present the general framework for modeling, and the details of the optics- and detector-related effects are described in the following sections.

If the brightness of the coronal scene in a particular passband is given by $B_{\mathrm{Cor}}$ and is expressed in [photon $\mathrm{s}^{-1} \mathrm{~cm}^{-2} \mathrm{sr}^{-1}$ ], then the number of photons $N$ coming to each pixel can be calculated from geometrical optics,

$N=B_{\text {Cor }} \cdot T_{\mathrm{f}} \cdot \mathrm{d} S_{\text {pix }} \cdot A p \cdot t_{\text {exp }}$,

where $T_{\mathrm{f}}$ is the dimensionless transmission of the optical system in the passband, $\mathrm{d} S_{\text {pix }}\left[\mathrm{sr}^{2}\right]$ is the solid angle of a pixel, $A p\left[\mathrm{~cm}^{2}\right]$ is the entrance aperture area, and $t_{\exp }[\mathrm{s}]$ is the exposure time.

The brightness can also be expressed in other physical units, such as [erg s${ }^{-1} \mathrm{~cm}^{-2} \mathrm{sr}^{-1}$ ] or [ $\left.\mathrm{W} \mathrm{m}^{-2} \mathrm{sr}^{-1}\right]$, by taking the photon energy $\varepsilon=\frac{h c}{\lambda}$ into account ( $h$ is Plank's constant, and $c$ is the speed of light). Our choice of using photons is dictated by the simplicity of the expressions for the detector sensitivity and for the shot noise of photons.

The photons are registered by the detector and converted into digital numbers (DN). The detector sensitivity $S\left[\mathrm{DN}\right.$ photon $\left.{ }^{-1}\right]$ can be expressed as a product of quantum efficiency $\mathrm{QE}_{\mathrm{f}}$ (dimensionless), and gain $g\left[\mathrm{DN}\left(\mathrm{el}^{-}\right)^{-1}\right]$. The signal $I[\mathrm{DN}]$, as measured by the detector, is expressed as $I=N \cdot S$, and Eq. (1) transforms into

$I=B_{\mathrm{Cor}} \cdot A_{\mathrm{f}} \cdot t_{\mathrm{exp}}$,

where $A_{\mathrm{f}}=T_{\mathrm{f}} \cdot \mathrm{d} S_{\text {pix }} \cdot A p \cdot \mathrm{QE}_{\mathrm{f}} \cdot g$ is the telescope photometric sensitivity expressed in [DN photon $\left.{ }^{-1} \mathrm{~cm}^{2} \mathrm{sr}\right]$. The subscript $\mathrm{f}$ denotes the passband (see Appendix A for the definition of $B_{\mathrm{Cor}}$ and the telescope characteristics with consideration of spectral dependences), but this is omitted further in the text.

Characteristics such as optical transmission $T$ and detector sensitivity $S$, and thus the photometric sensitivity $A$, may vary across the detector area, in other words, across the FOV. We can therefore define for every pixel $[x, y]$ its own $A_{x y}$. We split it into an average (over FOV) sensitivity, and a flat-field $A_{x y}=A \cdot F_{x y}$. The flat-field $F_{x y}$ is a dimensionless factor with its average being 1 over the FOV. In order to avoid $[x, y]$ indices and express the formula for every pixel in the image, we use $2 \mathrm{D}$ arrays, written in bold. The position of a pixel within the array, or its indices $[x, y]$, denote its position on the detector. The value stored in that pixel represents its intensity. We extensively use array arithmetics, thus we obtain

$\mathbf{I}=\mathbf{B}_{\mathrm{Cor}} \cdot \mathbf{F} \cdot A \cdot t_{\mathrm{exp}}$.

Commonly assumed detector effects such as dark signal and bias (also known as offset, or offset in fixed pattern noise, FPN) can be expressed by the additional terms DC $\cdot g \cdot t_{\exp }+$ bias in Eq. (3), which transforms into the following:

$\mathbf{I}=\mathbf{B}_{\mathrm{Cor}} \cdot \mathbf{F} \cdot A \cdot t_{\exp }+\mathbf{D C} \cdot g \cdot t_{\exp }+\mathbf{b i a s}$.

\section{Input coronal images}

From the point of view of a user of the simulated data, the input coronal image $\mathbf{B}_{\text {Cor }}$ is a parameter. The software for the simulated data generation should be capable of producing a correct output image I for any (not even coronal) input image. The use of realistic input images is necessary to correctly assess the exposure time, signal-to-noise ratio $(\mathrm{S} / \mathrm{N})$, etc. Furthermore, in order to unify the approach and software for different filters, and to simplify inclusion of optical effects, the smart choice of units for the input data is important.

The main contributors to the white-light observations are the $\mathrm{K}$ and $\mathrm{F}$ corona (Billings 1966). The narrow-band filters, in contrast, are designed to increase the relative contribution of the spectral lines, or E corona, to the passband.

\subsection{WBF images}

To calculate the signal for the WBF filter of ASPIICS, we used the input coronal scene expressed in mean solar brightness (MSB). The conversion from MSB to physical units [photon $\mathrm{s}^{-1} \mathrm{~cm}^{-2} \mathrm{sr}^{-1}$ ] is quite straightforward for a passband, which is dominated by the continuum emission. For this purpose, the solar spectrum was multiplied with the spectral passband of the instrument, and the spectral passband over wavelength gives the actual value of MSB. For the WBF filter of ASPIICS, the value 
is $\mathrm{MSB}_{\mathrm{W}}=2.02 \times 10^{20}$ photon $\mathrm{s}^{-1} \mathrm{~cm}^{-2} \mathrm{sr}^{-1}$. We wish to stress the relative nature of the mean solar brightness: If the same coronal scene were to be observed with a similar instrument but with a different passband, for instance, two times wider, the coronal scene would have the same appearance in [MSB]. However, the number of photons coming to each pixel will be different, two times larger in our example.

In order to convert a relative, that is, expressed in [MSB], input coronal image $\mathbf{B}_{\mathrm{W}}$ into an image that is expressed in physical units, we multiplied the relative input image by $\mathrm{MSB}_{\mathrm{W}}$ : $\mathbf{B}_{\mathrm{Cor}}=\mathrm{MSB}_{\mathrm{W}} \cdot \mathbf{B}_{\mathrm{W}}$. The resulting array was further processed using Eq. (4).

We used two types of input images: A synthetic and a more realistic image. The realistic image was registered during a total solar eclipse.

The synthetic K-corona image was created using a 2D mask, denoting different structures in the corona, and setting the brightness of each pixel according to its height and type of coronal structure. The radial dependences were taken from Allen (1976), and the typical brightness range from $10^{-5} \mathrm{MSB}$ at heights of $\sim 1.01 R_{\odot}$ (equatorial streamers) to $10^{-10} \mathrm{MSB}$ at heights of $\sim 3 R_{\odot}$ (polar region during solar minimum). Further details are given in Shestov et al. (2019, hereafter SH19). The resulting synthetic image does not represent the real corona in terms of variety of the observed structures. However, it has the correct dynamic range and realistic radial decrease of brightness. The high contrast between the structures makes it convenient for an investigation of the optical properties of the telescope.

The eclipse K-corona image is based on eclipse observations performed on 11 August 1999 at Râmnicu Vâlcea, Romania. Further details are given in SH19.

The synthetic and the eclipse images were cropped to the proper FOV and were rescaled to $2048 \times 2048$ pixels. The images are given in Fig. 2. The logarithmic color scale is the same for both images. The horizontal and vertical profiles taken through the centers are shown in the bottom panels. The eclipse and synthetic images have an artificial empty central region and vignetting that occurs $<1.1 R_{\odot}$. The actual vignetted zone of ASPIICS is shown by the gray shading in the bottom plots, and the dotted line denotes the solar limb.

The two images have a comparable dynamic range and minimum and maximum intensities. The eclipse image, while being more realistic, has much lower contrast. The details in it might be revealed by additional image processing, such as correcting for exponential radial decay. In the synthetic image, the structures with minimum intensity (polar structures during solar minimum) and maximum intensity are annotated. The horizontal profile in the right part crosses a streamer and a region of solar minimum, and in the left part, it crosses the quiet equatorial region.

We intentionally use arcseconds (or pixels) for the horizontal axes here and throughout the whole manuscript. The angular size of the Sun changes during the year, whereas most of the optical effects have a constant position in angular coordinates.

The F-corona input image is based on the radial profiles from Koutchmy S. in Cox (2000). The radial profiles of the F corona are given in Fig. 2, and the corresponding coronal scene is created similar to the synthetic $\mathrm{K}$ corona.

\subsection{NBF images}

The main purpose of NBF simulated images is twofold: calculating a realistic signal in the images, and testing the processing, where we reveal the contribution of the E corona by subtracting the continuum emission obtained from WBF observations.
The spectral shape of the narrow-band filters was assumed to be Gaussian. For the sake of the pipeline unification, the optical effects were considered in terms of MSB. If the Thomson scattering were the only contributor to the NBF passbands, we could use the same approach as for the WBF with appropriate coefficients: $\mathrm{MSB}_{\mathrm{Fe}}=3.742 \times 10^{18}$ photon $\mathrm{s}^{-1} \mathrm{~cm}^{-2} \mathrm{sr}^{-1}$ for the Fe XIV passband, or $\mathrm{MSB}_{\mathrm{He}}=1.548 \times 10^{19}$ photon s${ }^{-1} \mathrm{~cm}^{-2} \mathrm{sr}^{-1}$ for the He I D3 passband. Then the coronal scene could be calculated as usual: $\mathbf{B}_{\mathrm{Cor}}=\mathrm{MSB}_{\mathrm{Fe}} \cdot \mathbf{B}_{\mathrm{W}}$ or $\mathrm{MSB}_{\mathrm{He}} \cdot \mathbf{B}_{\mathrm{W}}$

The approach is easily enhanced to include the $\mathrm{E}$ corona. We started with the same images $\mathbf{B}_{\mathrm{W}}[\mathrm{MSB}]$ as for the WBF filter. Then we included the contribution of the $\mathrm{E}$ corona, $\mathbf{B}_{\mathrm{W}}$ becomes $\mathbf{B}_{\mathrm{W}}+\mathbf{B}_{\mathrm{Fe}}$ or $\mathbf{B}_{\mathrm{W}}+\mathbf{B}_{\mathrm{He}}$, and finally, we multiplied by the corresponding values of the $\mathrm{MSB}$, either $\mathrm{MSB}_{\mathrm{Fe}}$ or $\mathrm{MSB}_{\mathrm{He}}$.

The energetics and spatial distribution of the E-corona scene depend on the filter: the emission of He I D3 line is produced in cool prominences, which are usually low-lying and of limited size. The strong emission of the Fe XIV line is observed in coronal streamers. To create $\mathbf{B}_{\mathrm{E}}$, we therefore used an approach similar to approaches in synthetic WBF K-corona images: we created 2D masks representing structures, that is, prominences or streamers. For the He I line, we used the brightness of $8000 \mathrm{~K}$ and $30000 \mathrm{~K}$ prominences from Jejčič et al. (2018). For the Fe XIV line, we used the radial dependence of the brightness of a helmet structure and a streamer from Kim (1997). Then we converted the brightnesses from [ $\mathrm{erg} \mathrm{s}^{-1} \mathrm{~cm}^{-2} \mathrm{sr}^{-1}$ ] into [photon $\mathrm{cm}^{-2} \mathrm{~s}^{-1} \mathrm{sr}^{-1}$ ] using the corresponding photon energies $\frac{h c}{\lambda}$, and we finally divided by $\mathrm{MSB}_{\mathrm{He}}$ or $\mathrm{MSB}_{\mathrm{Fe}}$. The obtained $\mathrm{E}$ corona scene were used along with $\mathbf{B}_{\mathrm{W}}$. They are shown in Fig. 3 along with the full images that show the E, K, and F corona. We did not take the additional continuum emission produced by the prominence in the He I D3 filter into account, because this is more than an order of magnitude smaller than the D3 line emission at the assumed temperatures (Jejčič et al. 2018).

\subsection{Polarized images}

The main purpose of simulated polarized images is also twofold: Calculating a realistic signal in the images, and using them to test the calculation of the polarization parameters, which initially is Stokes $I, Q$, and $U$, and afterward the polarized brightness and polarization angle.

To create the input polarized images $\mathbf{B}_{\psi}$, we used a different approach. Here $\psi$ denotes the orientation of the polarizer direction with respect to the $x$-axis of the image, and it can be 0 , 60 , or 120 . The approach is based on the approaches described in Decraemer et al. (2019). A global coronal density model was taken from Guhathakurta et al. (1996), Table 3B and 3C, which included a polar corona and a streamer (called a current sheet therein). Then we created a 3D numerical data cube of the coronal density within the proper FOV, and provided it as an input to the SCRaytrace code (Thernisien et al. 2004, 2006b). The SCRaytrace code converts the density into polarized brightness $\mathrm{pB}$ and total brightness B following the equations for Thomson scattering. Then we calculated the polarized images $\mathbf{B}_{\psi}$ from $\mathrm{pB}$ and $B$ using the equations from Billings (1966). If $\alpha$ is the angle tangent to the solar limb and $p=p B / B$ is the degree of polarization (or simply polarization), then from the Malus law, we obtain

$B_{\psi}=\frac{B}{2}(1.0+p \cos 2(\psi-\alpha))$.

We created the $2048 \times 2048$ images $\mathbf{B}_{0}, \mathbf{B}_{60}$, and $\mathbf{B}_{120}$ expressed in MSB, and used them as an input instead of $\mathbf{B}_{\mathrm{W}}$. 

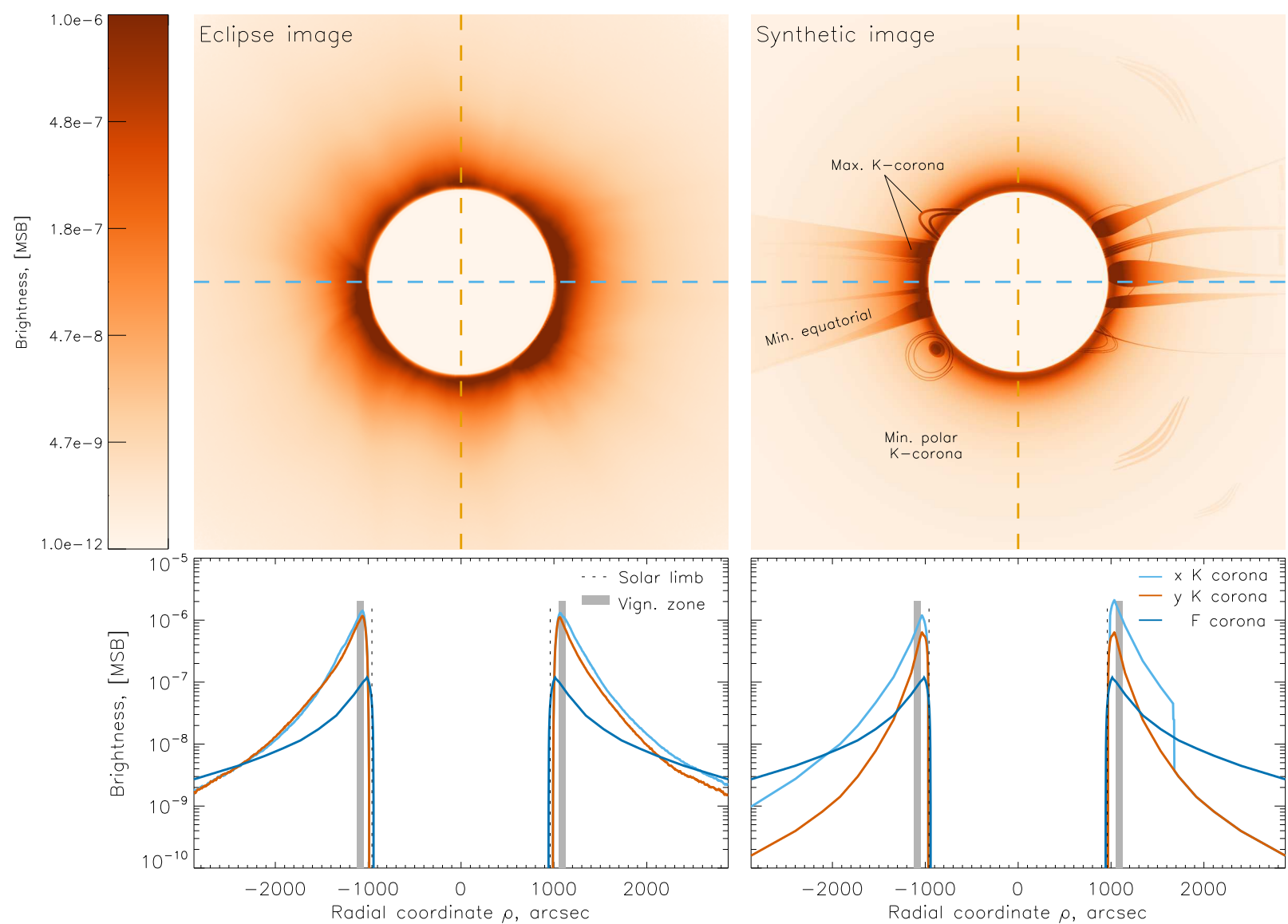

Fig. 2. Top: comparison of the eclipse (left) and the synthetic (right) images of the K corona. Bottom: intensity profiles of the K corona, taken along the dashed lines in the images, and of the F corona. The relative sizes of the Sun and of the vignetted zone in ASPIICS are given for reference.
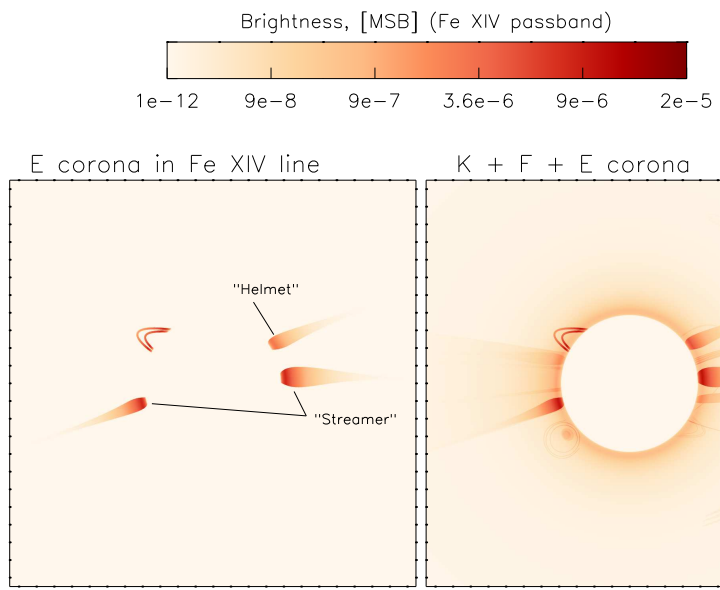
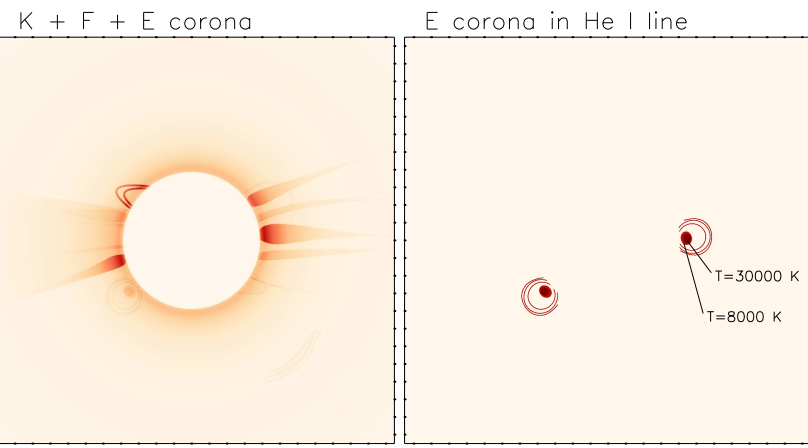

Fig. 3. Narrow-passband filter images. Left: E corona formed by the Fe XIV $530.45 \mathrm{~nm}$ line. Middle-left: total image composed of the K, F, and E corona observed in the Fe XIV passband. Middle-right: E corona formed by the He I D3 line $587.7 \mathrm{~nm}$. Right: total image composed of the K, F, and E corona observed in the He I D3 passband of ASPIICS. The logarithm color scales differ from the scale in Fig. 2.

Examples of the images and their radial and polar profiles are given in Fig. 4.

\section{Optical effects}

\subsection{Optical throughput}

The number of photons coming to a particular pixel of the detector is proportional to the geometrical factors: the pixel angular size $\mathrm{d} S_{\text {pix }}$, the aperture area $A p$, and the transmittance $T$ of the optical system. The aperture size is determined by the diameter of the Lyot stop, since it is conjugated to the entrance aperture and has smaller diameter. Its effective diameter is $97 \%$ of the diameter of the entrance aperture, hence $A p=\pi R_{\text {Lyot }}^{2}=18.47 \mathrm{~cm}^{2}$.

The solid angle of a pixel is calculated as $\mathrm{d} S_{\text {pix }}=\left(\frac{d x}{f_{\text {eff }}}\right)^{2}=$ $\left(\frac{10 \mu \mathrm{m}}{734.62 \mathrm{~mm}}\right)^{2}=1.853 \times 10^{-10} \mathrm{sr}^{2}$. The ratio of the pixel size to the 

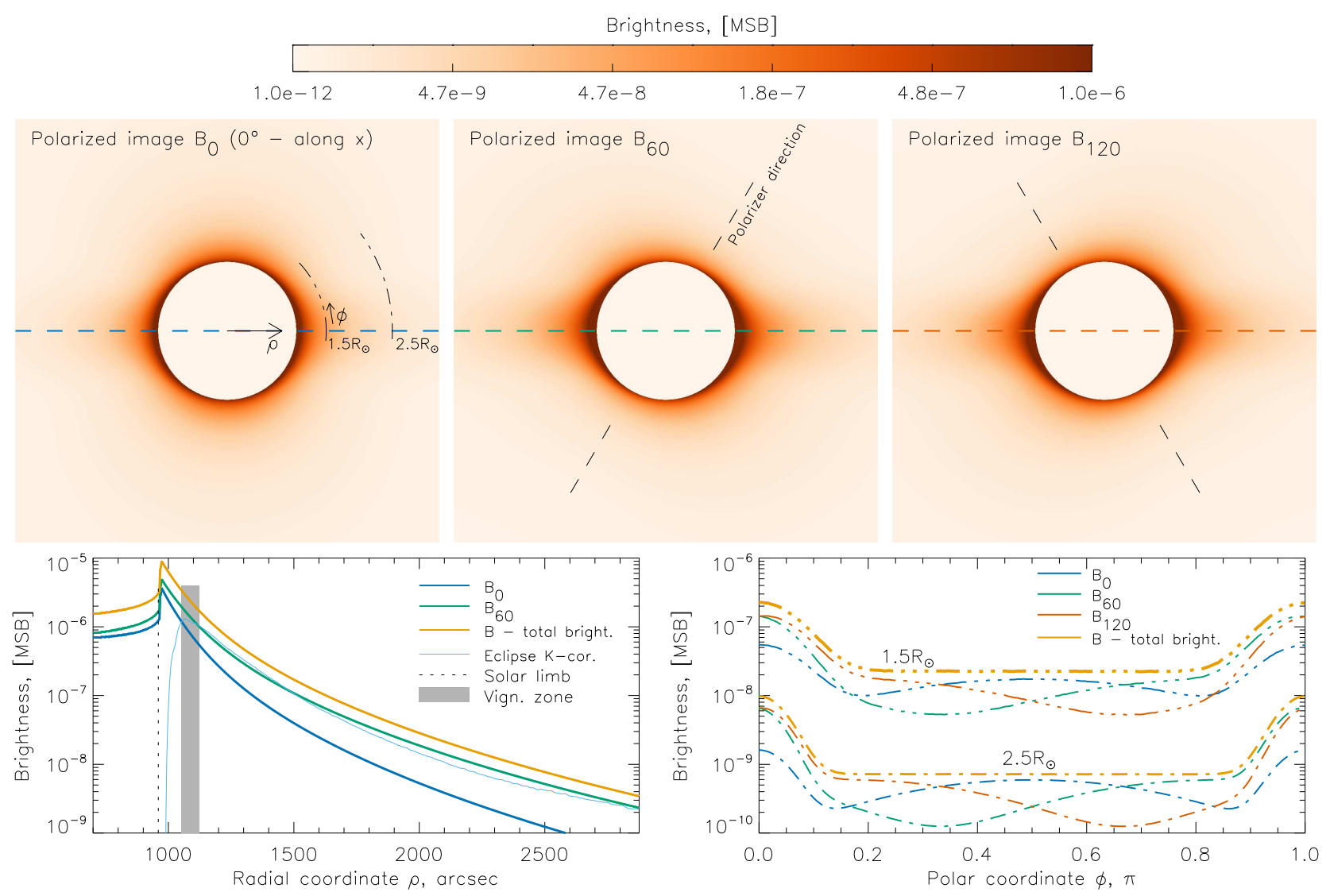

Fig. 4. Polarized images. Top panels: calculated images $\mathbf{B}_{0}, \mathbf{B}_{60}$, and $\mathbf{B}_{120}$ denoting the corona observed with different orientations of the polarizers. The logarithmic color scale corresponds to the scale from Fig. 2. Bottom panels: radial (left) and polar (right) profiles. For reference, we plot the total brightness $B$, as well as the brightness of the eclipse $\mathrm{K}$ corona, the solar limb, and the vignetting zone.

effective focal length $\frac{\mathrm{d} x}{f_{\text {eff }}}$ gives an angular width for a pixel of $1.36 \times 10^{-5}$ rad or 2.81 arcsec.

The optical transmittance is determined in the first place by the spectral filters of the telescope and the ND50\% filter in front of the detector, and then by the antireflective coatings of the lenses and their transparencies. The full transmittance depends on the filter: $T=0.393$ for the WBF, $T=0.369$ for the Fe XIV filter, $T=0.338$ for the He I filter, and $T=0.275$ for the polarized filters.

\subsection{Vignetting}

Obscuration of the entrance aperture of a coronagraph by the EO results in the decrease in photometric sensitivity of the instrument, or vignetting (Koutchmy 1988; Bayanna et al. 2011). Afterward, the IO inside the telescope additionally cuts out some light and reduces the sensitivity. Because the IO in ASPIICS is situated in a plane that is conjugated to the EO, we can consider the reprojected IO instead of the EO in the framework of geometrical optics (SZ18).

Aime et al. (2019) studied additional effect of the PSF degradation due to diffraction during aperture obscuration. The authors concluded that photometric sensitivity of the instrument degrades similar to the geometrical model, but spatial information about the coronal structure is completely lost in the inner vignetted zone where the vignetting is higher than $50 \%$.

In the simulated data pipeline we implemented the effect of geometrical vignetting. The minimum (the coronal light starts to reach the detector) and maximum (vignetting completely disappears) angles of vignetting are

$$
\begin{aligned}
& w_{\min }=\arctan \left(\frac{R_{\mathrm{IO}}^{\prime}-r}{z_{0}}\right)=17.551 \operatorname{arcmin} \approx 1.097 R_{\odot} \\
& w_{\max }=\arctan \left(\frac{R_{\mathrm{IO}}^{\prime}+r}{z_{0}}\right)=18.742 \operatorname{arcmin} \approx 1.171 R_{\odot},
\end{aligned}
$$

where $R_{\mathrm{IO}}^{\prime}=761.95 \mathrm{~mm}$ is the projected size of the internal occulter to the conjugated plane, $r=25 \mathrm{~mm}$ is the radius of the entrance aperture of the telescope, and $z_{0}=144348 \mathrm{~mm}$ is the distance to the conjugated plane (this is also the nominal value of the ISD).

In the registered images, the region of vignetting has the shape of a ring with a width of $\sim 1.2$ arcmin, or 25 pixels. The dependence of the unobscured aperture area on the field angle $\rho$ is depicted in Fig. 5. The length $h$ of the radius of the aperture, obscured by the occulter, is calculated as

$h=r-\tan \left(\rho-w_{\min }\right) \cdot z_{0}$.

The angle $\beta$ of the sector is

$\beta=2 \arccos (h / r)$.

Finally, the vignetting function, defined as optical transmittance and calculated as the ratio of the unobscured part to the full aperture area, is calculated as

$V(\rho)=\frac{\beta-\sin (\beta)}{2 \pi}$. 

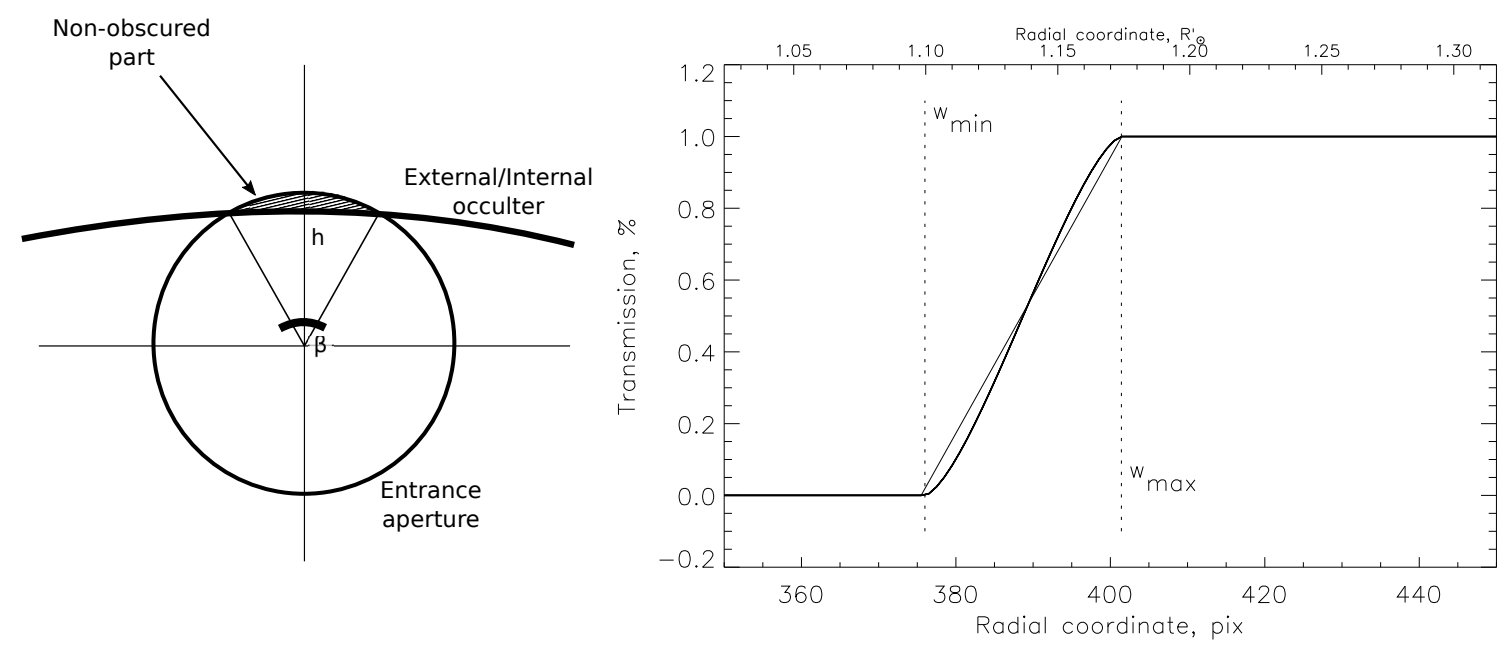

Fig. 5. Analysis of the vignetting effect. Left panel: obscuration of the entrance aperture by the internal occulter. Right panel: radial profile of the vignetting function shown with a thick line. The dashed lines denote the minimum $w_{\min }$ and maximum $w_{\max }$ vignetting angles, and the thin line denotes the linear interpolation. The upper scale is expressed in yearly average solar radius for comparison.

The obtained vignetting function is depicted in the right panel of Fig. 5 with a thick curve.

We created an array $\mathbf{V}$ with the vignetting in every pixel depending on its angular coordinate $\rho$, and multiplied the coronal scene: $\mathbf{B}_{\mathrm{W}} \cdot \mathbf{V}$.

\subsection{Diffracted light}

Diffraction plays a key role in white-light coronagraphy: the bright light from the solar disk propagates into the geometrical umbra of the EO. The diffracted light propagates inside the instrument and produces a rather severe stray-light pattern in the registered images. Many efforts have been devoted to minimizing its impact (e.g., Koutchmy 1988; Bout et al. 2000; Landini \& Mazzoli 2010; Landini et al. 2017b). Aime (2013) showed that because of the small overoccultation angle of the $\mathrm{EO}$, the amount of the diffracted light coming to the primary objective in ASPIICS will be essential. Later, RR17 enhanced the model and calculated the diffracted light on the detector through a Fourier-optics formalism. The authors confirmed that two additional diaphragms, the IO and the Lyot stop, can significantly reduce intensity of the diffracted light.

SZ18 further enhanced the RR17 model by taking various misalignments into account, such as the displacement of the IO from the optical axis, the deviation of the ISD from the nominal value, the mutual misalignment of the telescope, the EO, and the direction toward the Sun. The authors showed that any geometrical configuration of the Sun, the EO, and the telescope can be expressed as the sum of two misalignments. The first misalignment is the tilt of the telescope around the center of the entrance aperture, and the second misalignment, called solar shift below, is the displacement of the Sun from the original axis, which in reality can be achieved by a simultaneous tilt of the EO and the telescope as a rigid body.

The model from SZ18 computes the intensity of the diffracted light in terms of the mean solar brightness. The conversion into the number of photons is performed only later, using an approach similar to Eq. (1). For the purposes of generating simulated images, it is straightforward to consider the diffracted light as an additional light source $\mathbf{B}_{\text {Diff }}[\mathrm{MSB}]$ in the plane of sky. Then it is summed with the coronal scene $\mathbf{B}_{\mathrm{W}}$ and is treated in the same way as the coronal scene, that is, by applying Eq. (4).
Another advantage of this choice is connected with the necessity of rescaling: the diffraction calculations are sensitive to the numerical sampling, therefore it is desirable to use larger arrays, for instance, of $4096 \times 4096$ or more. The FOV and pixel angular size in the diffracted images depend on the numerical parameters and do not coincide with those of the simulated images. After the calculation, we therefore cropped the diffracted images to the desired FOV and rescaled them to the pixel plate scale 2.81 arcsec. Using the MSB does not require modifying the signal inside the pixels. This modification is required when the diffracted scene is expressed in photons per pixel because a virtual four times smaller pixel would receive four times fewer photons.

We stress that $\mathbf{B}_{\text {Diff }}$ reflects the diffracted scene as registered by the detector. The actual diffracted light entering the telescope will have much higher brightness and slightly different 2D distribution. In contrast with the coronal scene, the diffracted light does not experience optical vignetting: the diffraction effect is the propagation of light into the region of the geometrical umbra and the vignetting zone.

Examples of diffraction scene $\mathbf{B}_{\text {Diff }}$ calculated for the current optical design with $r_{\mathrm{IO}}=1.748 \mathrm{~mm}$ and $R_{\mathrm{Lyot}}=0.97 R_{A}$ and for different combinations of tilt and solar shift are given in Fig. 6 (a constant ISD value $z_{0}=144348 \mathrm{~mm}$ and solar angular size $R_{\odot}=16$ arcmin were used). The effect of the solar angular size on the diffracted scene is partially covered in Sect. 5.5.

\subsection{Ghost light}

Galy et al. (2019) performed a preliminary analysis of ghost light in ASPIICS with a simplified symmetrical corona as input. The authors showed that the ghost image was mainly produced by two additional reflections of the incoming light: initially by the detector (reflectivity $R \sim 15 \%$ ), and later by neighboring optical surfaces, that is, by the detector glass, the filters, and the lenses of $\mathrm{O} 3(R \sim 0.5 \%)$. These reflections, however, redistribute the intense signal from the inner corona higher up, which causes the ghost contribution to become significant in these regions. In order to reduce the relative effect of the ghost light, an absorptive ND50\% filter was introduced in the optical design instead of the transparent detector glass. The ND50\% filter reduces the intensity of normal light (coronal scene, diffracted light scene) 


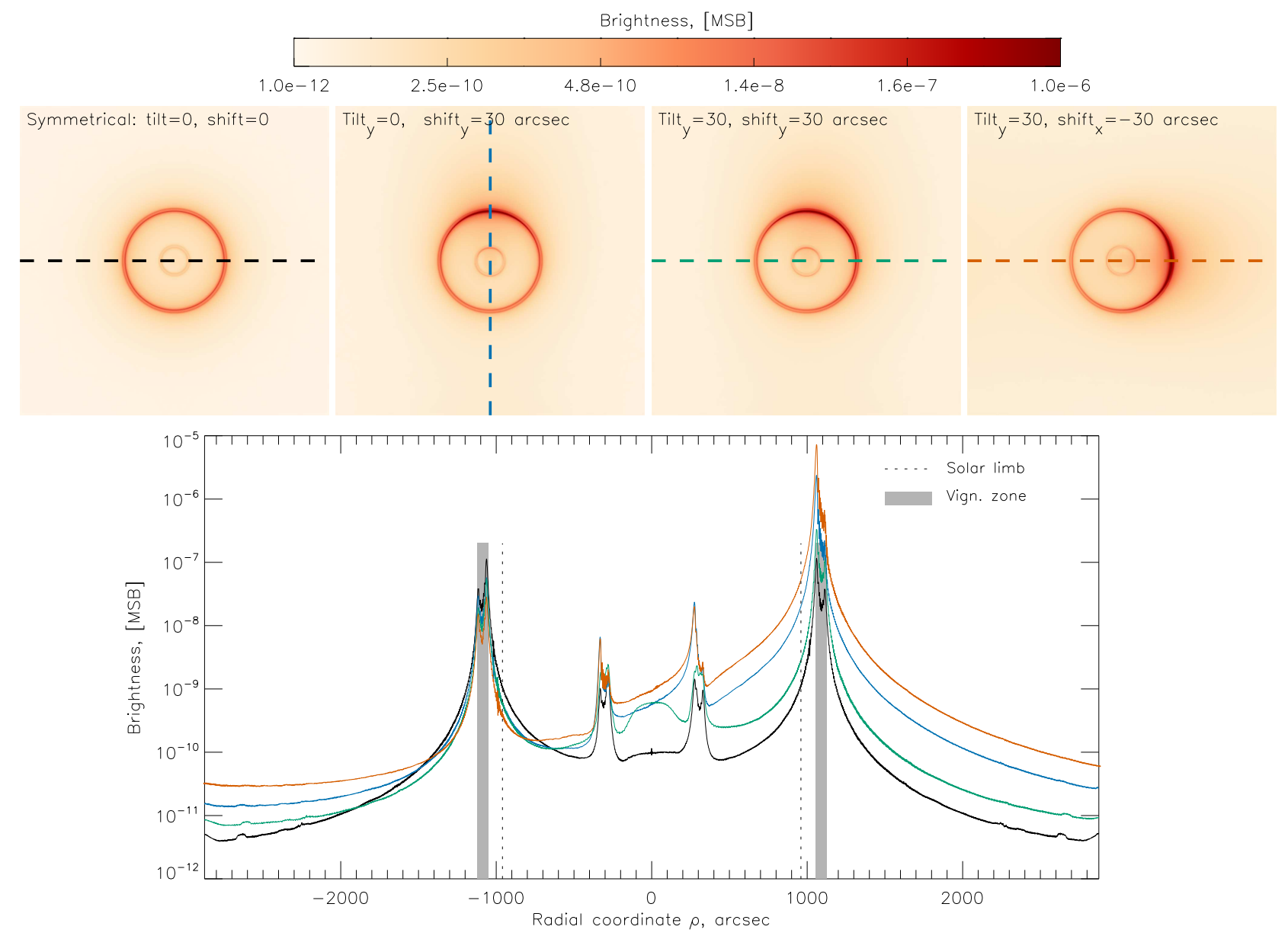

Fig. 6. Comparison of the diffracted light images calculated for various misalignments, while keeping the ISD $z_{0}=144348 \mathrm{~mm}$ and solar angular size $R_{\odot}=16$ arcmin constant. The panels from left to right show a symmetrical configuration, a solar shift of 30 arcsec along the vertical direction, the same solar shift and the telescope tilt around the vertical axis (in the image the effects occur in the $y$ - and $x$-directions), and finally, the solar shift in the horizontal direction and the tilt around the vertical axis (constructive effect along the $x$-axis in the image). The logarithmic color scale is slightly different from that in Fig. 2. The profiles in the images are given in the bottom panel, the vignetted zone is given by the shaded area, and the solar limb is denoted by dotted lines.

by a factor of 2 , but it reduces the intensity of the ghost scene by a factor of 8 .

A more advanced investigation of the ghost light was performed in SH19. The authors investigated the behavior of ghost contributors that were produced by every pair of optical surfaces (confirming the main results of Galy et al. 2019) and created a model for calculating a ghost scene for an arbitrary input image. Because of the prevailing contribution of the coronal and the diffracted scene to the optical scene, the ghost scene was calculated based on the sum of the two: $\mathbf{B}_{\mathrm{G}}=\mathcal{G}\left(\mathbf{B}_{\mathrm{W}} \cdot \mathbf{V}+\mathbf{B}_{\text {Diff }}\right)$. The calculations can be performed in any units because the ghost scene is expressed in the same units as the input image. While the ghost light is formed inside the telescope, we considered the ghost scene to be in the plane of sky in order to unify the pipeline. Therefore we added $\mathbf{B}_{\mathrm{G}}$ to the coronal and diffracted light scene: $\mathbf{B}_{\mathrm{W}} \cdot \mathbf{V}+\mathbf{B}_{\text {Diff }}+\mathbf{B}_{\mathrm{G}}$. Because the ghost light is formed by the optics after the IO, the ghost scene does not experience vignetting. We compare the ghost scene with other optical signals in Sect. 5.8.

\subsection{Scattered light}

The scattered light was studied in detail in SH19. The authors used a ray-tracing approach with $\mathrm{ABg}$ prescription (Pfisterer
2011) of light scattering by the lens surfaces. They took the estimated values of the $\mathrm{ABg}$ parameters of every lens (Galy et al. 2019), and calculated the results of the scattering by different optical surfaces on the detector. The important property of the scattering is that the $2 \mathrm{D}$ pattern that forms on the detector significantly depends on the particular optical element and its position within the optical layout. For example, if a single planeparallel wave falls on the entrance aperture, the scattering by the PO forms an almost uniform illumination on the detector, whereas the scattering of the same wave by the field lens O2 forms a 200 pixel halo that is coaxial with the incoming beam (see Fig. 11 in SH19). During its propagation through the optical system, the diffracted light experiences similar scattering. Furthermore, the amount of the diffracted light that falls on the PO is significantly higher than on other surfaces because the diffracted light is efficiently blocked by the IO. SH19 showed that the scattering of the diffracted light on the PO, despite the sufficiently high optical quality of the lens, causes the prevailing contribution to the scattering. Scattering of the diffracted light by other lenses, as well as scattering of the coronal light, do not play an essential role.

We also tried to extend the model of Rougeot et al. (2019) and included the effects of misalignments, such as solar shift and the telescope tilt (following the approach of SZ18). 


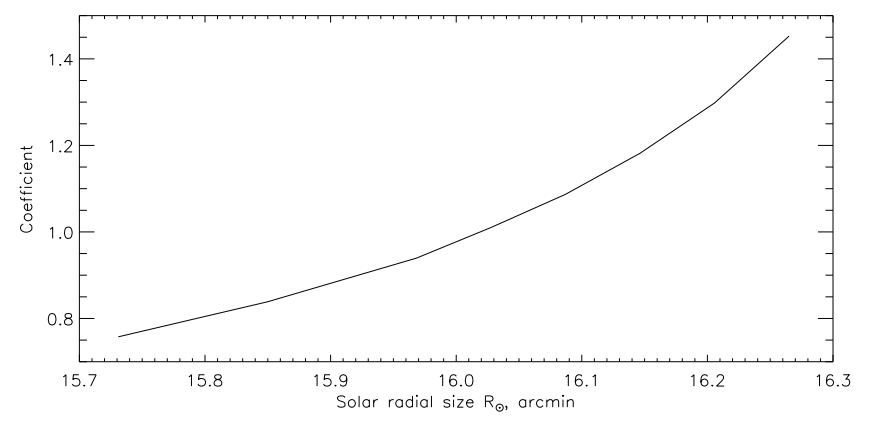

Fig. 7. Dependence of the intensity of the diffracted light incident on the $\mathrm{PO}$, and intensity of the diffracted light that is scattered by the PO on the solar angular size $R_{\odot}$. The values are normalized to the $R_{\odot}=16$ arcmin.

We found that misalignments did not change the scattered signal.

SH19 concluded that the diffracted signal with scattering on the PO can be considered as the sum of two components: the diffracted light without scattering, and a smooth component representing the scattering. These properties confirm that scattered light loses the coherent and diffractive properties (propagation to the regions of the geometrical umbra) during the scattering.

The effect of the scattering by the PO can be considered as follows: the surface of the PO acts as a light source, and its brightness depends on the surface roughness of the PO. The remaining optical system produces a strongly defocused image of the PO on the detector.

Numerical models for the diffracted light show that the amount of light incident on the PO (and ultimately reaching the detector) depends on the angular size of the Sun $R_{\odot}$. Obviously, $R_{\odot}$ also changes the amount of the diffracted light that is scattered by the PO. The dependence of the total intensity of the diffracted light incident on the PO during the seasonal change of $R_{\odot}$ from $15.73^{\prime}$ to $16.26^{\prime}$ is presented in Fig. 7 , and the intensity of the scattered light has the same dependence on $R_{\odot}$.

In contrast with the diffracted light, the scattered light on the detector does not depend on the in-orbit coalignment or tilt of the telescope. In the entrance aperture plane the intensity of the diffracted light enhances very slightly in the umbra region with the radial distance from the axis; see Fig. 4 in Landini et al. (2017a), Fig. 5 in RR17, and Fig. 1 in Rougeot et al. (2018). Thus the PO will receive the same amount of the incoming diffracted light, regardless of the relative position of the satellites and the Sun, if the entrance aperture is kept in the region of the geometrical umbra.

In order to calculate the scattered light on the detector, we therefore used a 2D pattern of scattering calculated for $R_{\odot}=$ 16 arcmin and modified its intensity according to Fig. 7. The scattered light was calculated in the same units as the diffracted light, thus it can be considered as a plane-of-sky source along with the other optical components, and the input optical scene becomes $\mathbf{B}_{\mathrm{W}} \cdot \mathbf{V}+\mathbf{B}_{\text {Diff }}+\mathbf{B}_{\mathrm{G}}+\mathbf{B}_{\mathrm{Sc}}$.

An example of a scattered light scene for the solar angular size $R_{\odot}=16$ arcmin is given in Sect. 5.8.

\subsection{Point spread function of the telescope}

The PSF of a telescope is a profile of the beam in the detector plane when it is illuminated by a plane-parallel beam. In general, the PSF may include the effect of the nonideal profile of optical surfaces, diffraction due to a finite aperture, effects such as ghost and scattering light intrinsic to the optical instrument, etc. In our model the effects of ghost and scattered light are considered separately, therefore the PSF is determined by the ray-tracing and diffraction performance of the optical system. According to the current optical design, the PSF of the telescope is diffraction limited and is slightly larger than 1 pixel (Galy et al. 2015).

Whereas for the analysis of coronal structures, implementing such a narrow PSF might not be necessary, it will be interesting for understanding how stars and low-contrast features will look in the ASPIICS images. In order to mimic such a narrow PSF, we applied a spatial filtering in the Fourier space, using a kernel with a modulation transfer function (MTF) of 0.5 at the highest spatial sampling of 50 lines $\mathrm{mm}^{-1}$. This MTF corresponds to the diffraction-limited PSF. The convolution is obviously a linear operation. Because the diffracted light is calculated including the effect of finite apertures, it should not be processed with the current Airy-PSF.

\subsection{Star field}

Stars are often used to refine the orientation and photometric sensitivity of solar coronagraphs (Gardès et al. 2013; Colaninno \& Howard 2015; Thompson 2018). A preliminary analysis shows that because of the small FOV of ASPIICS, only a relatively small number (about five or fewer) of bright stars with a magnitude $V<9$ will be present in the FOV, with an occasional presence of up to ten stars. Furthermore, registering the signal from individual stars in the images might be challenging in view of other light sources.

In order to unify the whole pipeline, we created a star field scene $\mathbf{B}_{\text {Star }}$, where the stars were placed in the proper positions. Their signal was expressed in [MSB]. This star field was used along with other light sources in the pipeline.

To calculate the position of stars within the ASPIICS FOV, we used the following approach: we placed the telescope at the center of Earth and assumed its orientation as follows: the $z$ axis was strictly toward the Sun center, and the $x$ - and $y$-axes coincided with the pixel coordinates. We used the HIPPARCos catalog (ESA 1997; Perryman et al. 1997), and initially convert the coordinates of every star from right ascension and declination $\left(r_{A}, \delta\right)$ into a Cartesian unit vector to remain in the original Equatorial frame of reference,

$\left(\begin{array}{c}\xi \\ \eta \\ \gamma\end{array}\right)=\left(\begin{array}{c}\cos r_{A} \sin \delta \\ \sin r_{A} \sin \delta \\ \cos \delta\end{array}\right)$

By a series of rotations, we then obtain the stellar coordinates $(x, y, z)$ in the telescope-related reference frame,

$\left(\begin{array}{l}x \\ y \\ z\end{array}\right)=\operatorname{Rot}_{1}\left(\delta_{\odot}^{\prime}-90\right) \cdot \operatorname{Rot}_{3}\left(r_{A \odot}^{\prime}-90\right) \cdot \operatorname{Rot}_{1}(\varepsilon) \cdot\left(\begin{array}{l}\xi \\ \eta \\ \gamma\end{array}\right)$,

where $\operatorname{Rot}_{i}$ denotes the rotation-matrix around the $i$ th coordinate, $\varepsilon=23.439$ is the Earth obliquity, $\left(r_{A \odot}^{\prime}, \delta_{\odot}^{\prime}\right)$ are the modified coordinates of the Sun in the reference frame corrected for the obliquity, and were obtained from right ascension $r_{A \odot}$ and declination $\delta_{\odot}$ of the Sun. In both cases, corrections for obliquity are needed to coalign the detector with the ecliptic north so that the stars travel horizontally in the images. Finally, the stars were projected into the image $\left(x_{\mathrm{det}}, y_{\mathrm{det}}\right)$ using $f_{\mathrm{eff}}, \mathrm{d} x$ and a simple projection (i.e., neglecting optical distortion),

$\left(\begin{array}{l}x_{\mathrm{det}} \\ y_{\mathrm{det}}\end{array}\right)=\left(\begin{array}{l}x_{C} \\ y_{C}\end{array}\right)+f_{\mathrm{eff}} / \mathrm{d} x \cdot\left(\begin{array}{l}\arctan x / z \\ \arctan y / z\end{array}\right)$ 


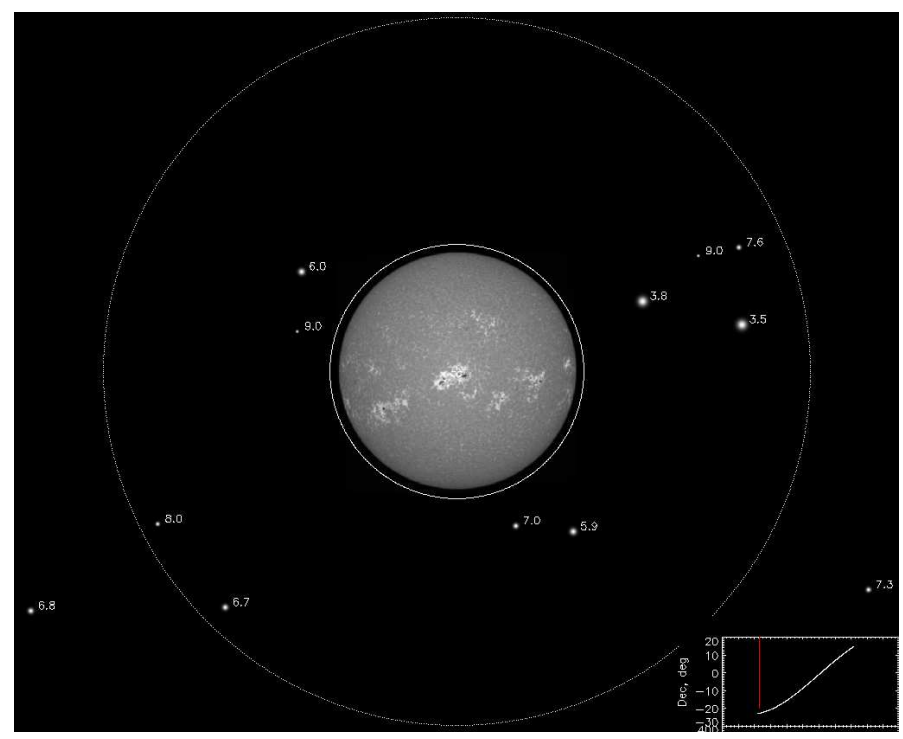

Fig. 8. Example of the star appearance in the ASPIICS FOV. The stars are calculated for 3 January 2015 with the telescope placed at the center of Earth and pointed to the center of the Sun. The approximate ASPIICS FOV is denoted by dotted circles. The star magnitude is given. The size of stars in the image denotes their brightness. The solar image in Ca II is shown for reference.

These transformations were applied to all the $\sim 120000$ stars in the HIPPARCos catalog, and only the stars within the FOV were kept. An example of the star field that would be observed by ASPIICS on 3 January 2015 is given in Fig. 8. The chosen date is the best date throughout the year, with the maximum number of stars present in the FOV of the ASPIICS. The approximate ASPIICS FOV is denoted by dotted circles in the figure, and the star magnitude is annotated and is also denoted by size of stars. The image of the solar disk observed in the CaII line is artificially included for reference (observed by the Uccle Solar Equatorial Table (USET) ${ }^{1}$, Royal Observatory of Belgium, Brussels).

In order to convert the star magnitude into MSB, we neglected the difference in spectral index and used the following transformations:

$B_{j}=\Omega_{\odot} \cdot 2.512^{-V_{j}-26.74}$,

where $\Omega_{\odot}=\frac{1}{\pi R^{2}}$, and solar magnitude is -26.74 .

All the stars from the FOV were placed in the final image $\mathbf{B}_{\text {Star }}$, but because the coordinates $\left(\begin{array}{l}x_{\mathrm{det}} \\ y_{\mathrm{det}}\end{array}\right)$ can be fractional, a small PSF was applied to preserve the full signal of the star. We later convolved the whole image with the PSF of the telescope.

\subsection{Comparison of optical effects}

We compare the different optics-related effects is given in Fig. 9. The comparison shows the dominant contribution, at least 2-3 orders of magnitude, of the coronal light in the main part of the FOV of ASPIICS. The predominance of the coronal signal persists even for the dimmest coronal signal, such as coronal holes. The diffraction gives the essential contribution only in the innermost zone, below $\sim 1.3 R_{\odot}$, where depending on the coalignment, it can have a comparable or dominant contribution.

Nevertheless, the diffracted light contribution is significantly lower on average than that of the corona. This reduction in

\footnotetext{
1 http://WwW.sidc.be/uset/
}

diffracted light throughout the whole FOV of ASPIICS was achieved by increasing the sizes of the IO and the Lyot stop. It is interesting to note the extremely low fluxes expressed in photons of the stray-light contributors at larger heights. They are low even for the most sensitive spectral channel of ASPIICS, and for a relatively long exposure time $t_{\exp }=1 \mathrm{~s}$.

\section{Detector-related effects}

Our general model for the detector behavior is the following: In each pixel, the light flux is accumulated during exposure time $t_{\text {exp }}$ and the total number of photons $N$ is converted with the quantum efficiency QE into the photo-electrons in the bulk of the silicon. At the same time, the dark current produces a dark signal in the pixel, with the full collected charge DC $\cdot t_{\text {exp }}$. Then the total accumulated charge is converted into voltage and digitized by the analog-to-digital converter (ADC), with the total conversion factor, or gain, $g\left[\mathrm{DN}\left(\mathrm{el}^{-}\right)^{-1}\right]$. The process of charge readout and digitization can be nonlinear, which in turn can be the result of several nonlinear processes (conversion into voltage, digitization, etc). However, in order to simplify the model, we treat the whole process as two steps: the initially nonlinear transfer function $\mathcal{L}()$ implements nonlinearity and modifies the collected charge, then the fixed electronic gain $g$ produces [DN]. In addition, some positive voltage (bias) is added to the signal before the digitization, but for simplicity, we express it in [DN] and consider it as an additional term after digitization. The registered signal $\mathbf{I}_{\mathrm{nf}}[\mathrm{DN}]$ can be expressed as follows:

$\mathbf{I}_{\mathrm{nf}}=\mathcal{L}\left(\mathbf{N} \cdot \mathbf{F} \cdot \mathrm{QE}+\mathbf{D C} \cdot t_{\mathrm{exp}}\right) \cdot g+\mathbf{b i a s}$,

where the index $\mathrm{nf}$ denotes the noise-free signal because it does not include the main sources of noise so far.

Our model implies that the flat field $\mathbf{F}$ explicitly includes variation over the FOV of the quantum efficiency QE and may include variation of the optical transmittance $T$, whereas the gain $g$ and the nonlinear transfer function $\mathcal{L}()$ are constant throughout the FOV (we discuss the limitations of this assumption in Sect. 9). This in turn allows us to assume that only QE and $\mathbf{F}$ depend on the passband, whereas the gain $g$ and other detector characteristics, such as bias, readout noise, and the position of hot pixels, do not.

For the purpose of the simulated data pipeline, we take some characteristics, gain, readout noise, and bias from Piqueras $(2013)^{2}$, in which the detector of the Polarimetric and Helioseismic Imager (PHI) instrument (Solanki et al. 2020) on board Solar Orbiter were analyzed. The PHI instrument has a detector and camera of the same type as ASPIICS. The main characteristics presented in Piqueras (2013) are also summarized in Table 1.

The quantum efficiency QE depends on the passband: for the $\mathrm{WBF}, \mathrm{He} \mathrm{I}$ and the polarizers $\mathrm{QE}=0.65$, and for the Fe XIV $\mathrm{QE}=0.61$.

The gain is taken as $g=0.119 \mathrm{DN}\left(\mathrm{el}^{-}\right)^{-1}$ (Piqueras 2013).

Two-dimensional maps of the dark current, bias, and the flat field are presented in Fig. 10. For the flat field and the dark current, we took artificial 2D patterns and adjusted them to realistic values from Piqueras (2013). The four-stripe structure of the bias was taken from Piqueras (2013) and is connected with the electronics design of the camera. The color tables are different to reflect the different units.

The saturation of the detector is determined by its full well depth of $\sim 10^{5} \mathrm{el}^{-}$and by the ADC output size of 14 bits. Strong

\footnotetext{
2 Available online here.
} 


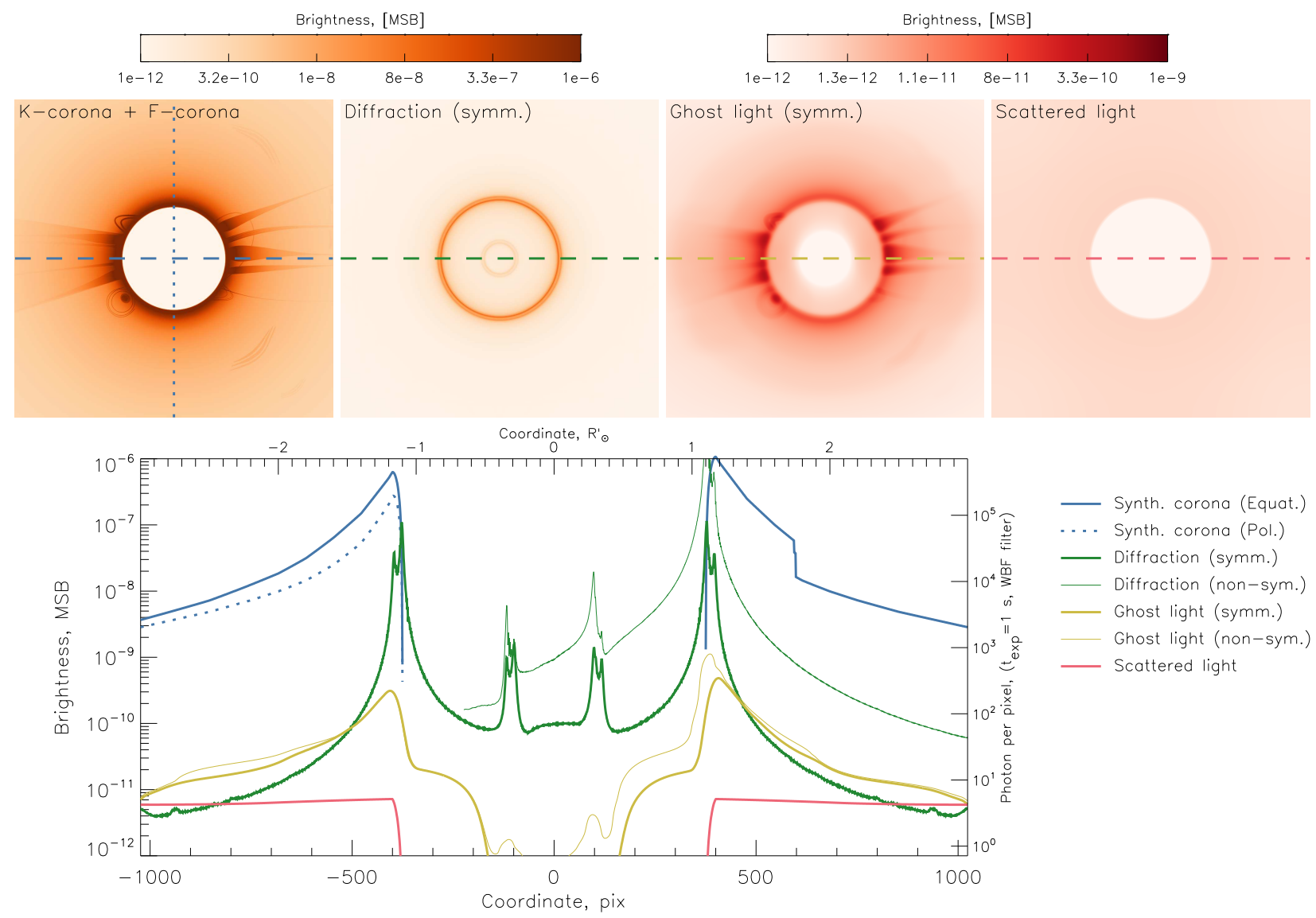

Fig. 9. Comparison of different optics-related effects. The images represent the synthetic corona, the diffracted light, the ghost light and the scattered light. The diffracted light is calculated for a symmetrical configuration. The brightness of the images is expressed in MSB, the color scale is the same for coronal and diffraction scenes, and another one for the ghost light and scattered light scenes. Bottom panel: horizontal profiles from the images are denoted by the blue, the green, the yellow, and the red solid lines, correspondingly, while the blue dotted line denotes vertical profile from the coronal scene, which corresponds to the polar corona. The thin green line represents diffraction for the worst non-symmetrical configuration, with both telescope tilt and solar shift (right panel in Fig. 6), the thin yellow line represents the ghost light calculated for this nonsymmetrical configuration. The left vertical axis is expressed in MSB, whereas the right axis is expressed in photon per pixel, which is obtained by applying Eq. (1) using $t_{\text {exp }}=1 \mathrm{~s}$ and taking into account $\mathrm{MSB}_{\mathrm{W}}$.

Table 1. Main characteristics of the detector measured in Piqueras (2013).

\begin{tabular}{lcc}
\hline \hline Name (Original name) & Value & Units \\
\hline Gain (Conversion gain) & 0.119 & $\mathrm{DN} / \mathrm{e}^{-}$ \\
Full well charge & 11700 & $\mathrm{DN}$ \\
Read-out noise & 6.013 & $\mathrm{DN}$ \\
Dark current & $20-60$ & $\mathrm{e}^{-} / \mathrm{s}$ \\
Bias (Offset FPN) & $50-800$ & $\mathrm{e}^{-}$ \\
\hline
\end{tabular}

signals will experience nonlinearity due to the saturation of the well depth and will additionally be clipped to the maximum possible value of $2^{14}-1=16383 \mathrm{DN}$. Because we are interested in analyzing images and understanding the telescope performance, we modified the assumed detector nonlinearity and did not apply any clipping connected with the ADC saturation. Instead, we compared the calculated signal with the maximum value of $16383 \mathrm{DN}$.

The nonlinearity of contemporary $\mathrm{Si}$-detectors usually does not exceed a few percent. However, especially in view of the currently foreseen multi-exposure regime of ASPICS, its modeling and correction during scientific data processing is of great importance. In the framework of preparing the Metis coronagraph (Antonucci et al. 2020), the nonlinearity of a similar detector has been measured recently (B. Inhester \& L. Teriaca, priv. comm.). The nonlinearity function, defined as the ratio of the measured and the linear response minus one, did not exceed $0.5 \%$ in most of the dynamic range and reached $-2 \%$ near the full well-depth level (see Fig. 11). Because we are going to preserve a large dynamic range of the simulated data, we smoothly extended the nonlinearity function to higher argument values and set it to zero. This resulted in a linear response of the detector at higher values of the input signal. The result is depicted in Fig. 11. The nonlinearity function is shown in the top panel, and the nonlinear transfer function $\mathcal{L}()$ in the bottom panel.

Hot pixels are implemented through a fixed list, and each pixel from this list was assigned to the maximal value. The readout noise was taken as $\sigma_{\mathrm{RN}}=6.013 \mathrm{DN}$ (Piqueras 2013).

\section{Implementation of noise and complete simulated data pipeline}

We then assembled all the effects into a complete simulated data pipeline that converted $\mathbf{B}_{\mathrm{W}}$ into $\mathbf{I}$. We denote by $\mathbf{B}_{\text {tot }}$ the resulting optical scene expressed as [MSB] 

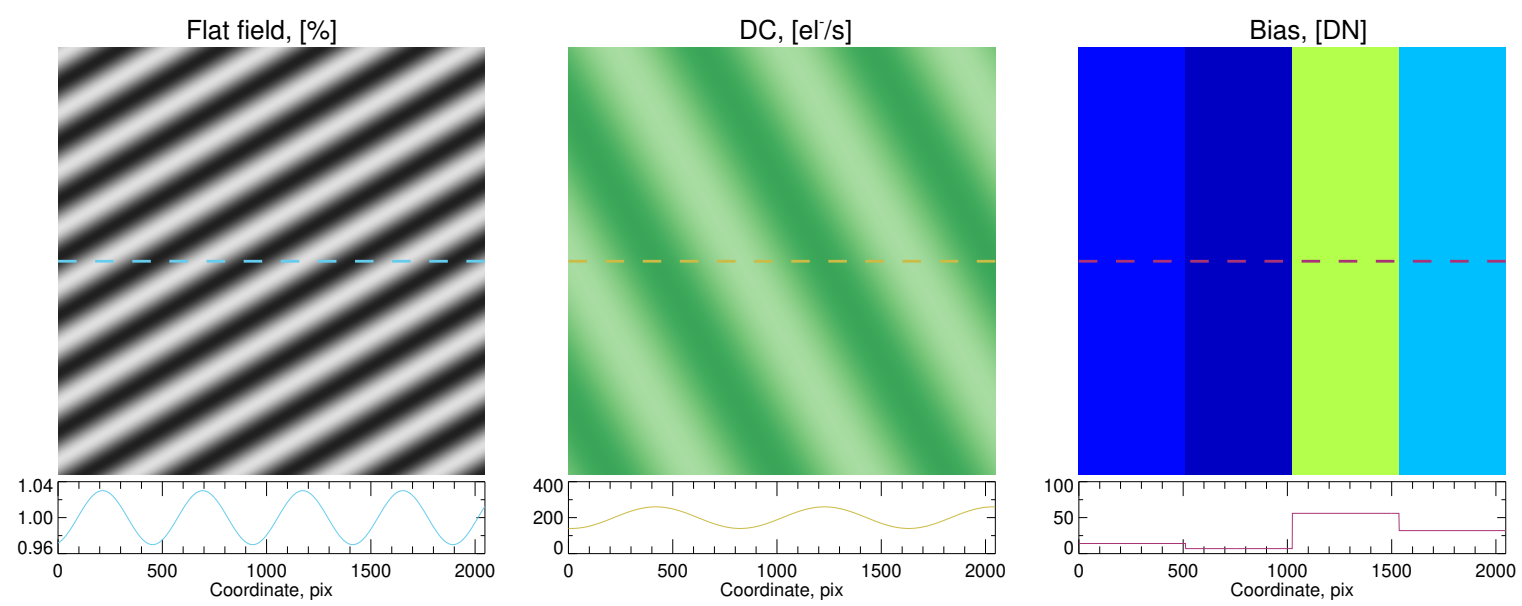

Fig. 10. Adopted 2D maps of the flat field (left panel), dark current (middle panel), and bias (right panel). Below each panel we show a horizontal profile through the center of the image. The $y$-axis units of each panel are designated at the top of the images.

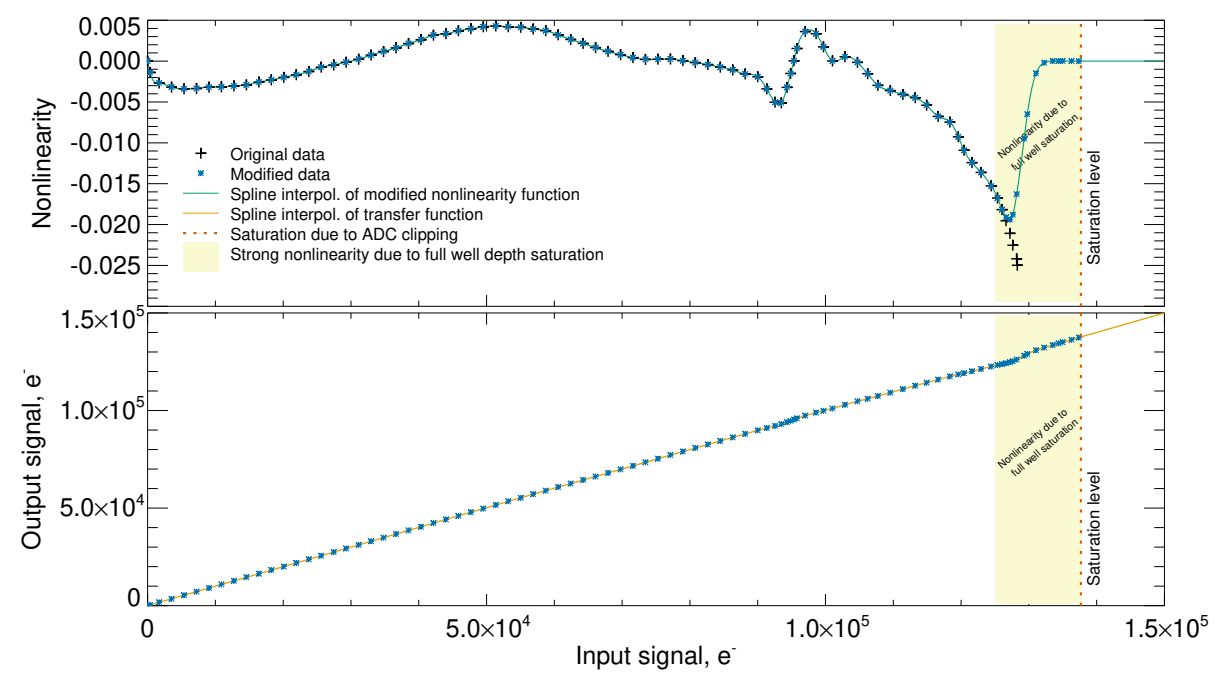

Fig. 11. Adopted detector nonlinearity. Top panel: nonlinearity function, defined as the ratio of the measured response to a linear response minus one. Bottom panel: nonlinear transfer function $\mathcal{L}()$. Pluses denote originally measured data, asterisks denote modified data, and solid lines denote spline interpolation. The vertical dotted lines denote the clipping by $\mathrm{ADC}$ at $2^{14}[\mathrm{DN}]$, and the filled area denotes the strong nonlinearity due to the full well depth (see details in the text).

$$
\mathbf{B}_{\mathrm{tot}}=\mathbf{P S F}\left(\left(\mathbf{B}_{\mathrm{W}}+\mathbf{B}_{\mathrm{Star}}\right) \cdot \mathbf{V}+\mathbf{B}_{\mathrm{G}}+\mathbf{B}_{\mathrm{Sc}}\right)+\mathbf{B}_{\mathrm{Diff}}
$$

An almost-complete simulated image $\mathbf{I}_{\mathrm{nf}}$ is obtained using the following processing chain:

$$
\begin{aligned}
\mathbf{I}_{\mathrm{nf}}= & \mathcal{L}\left\{\mathbf{B}_{\mathrm{tot}} \cdot \mathrm{MSB}_{\mathrm{W}} \cdot T \cdot \mathrm{d} S_{\mathrm{pix}} \cdot A p \cdot \mathbf{F} \cdot \mathrm{QE} \cdot t_{\mathrm{exp}}\right. \\
& \left.+\mathbf{D C} \cdot t_{\mathrm{exp}}\right\} \cdot g+\text { bias. }
\end{aligned}
$$

This formula represents the detailed version of Eq. (4). It is written for the mean values, without random noise.

In order to implement the effect of noise, we recall that there are various physical contributors of noise: the shot noise of arriving photons, the shot noise of dark electrons, the readout noise of the detector, and the effect of quantization, which is usually called ADC noise. Each of these noise contributors is considered to be independent of the others. The realization of the noise value in every particular pixel is independent of the other pixels and of its own values in the previous or the successive images. There are two general approaches to implement the effect of noise in the simulated data: either to calculate a realization of the noise of every physical contributor and sum the noisy components in Eq. (17), or when they are assumed to be Gaussian distributed, an analytical formula for the resulting noise is derived and its realization is added to the noiseless image $\mathbf{I}_{\mathrm{nf}}$ in Eq. (17). The first approach proved to be very efficient during the analysis of
ASPIICS performance because it allowed assessing the contribution of each physical mechanism to the final simulated data.

For the shot noise of photons, we assumed that the registered photo-electrons obey Poisson statistics. Thus, instead of an array in which the number of photo-electrons is registered in every pixel $\mathbf{M}=\mathbf{N} \cdot \mathbf{F} \cdot \mathrm{QE}$, we should use an array with a Poisson realization $\mathcal{P}(\mathbf{M})$ with the average (over a set of realizations) number $\mathbf{M}$ in each pixel. For practical reasons, we used Gaussian noise instead of Poisson, and $\mathbf{M}$ becomes $\mathbf{M}+\mathcal{R}^{2 \mathrm{D}} \cdot \sqrt{\mathbf{M}}$. The function $\mathcal{R}^{2 \mathrm{D}}$ produces a $2 \mathrm{D}$ array with normally distributed random values, with $\sigma=1$ in each pixel. Each invocation of $\mathcal{R}^{2 \mathrm{D}}$ gives a different realization with the desired statistics.

For the shot noise of dark electrons, we used the same approach, and the number of dark electrons DC $\cdot t_{\text {exp }}$ becomes $\mathbf{D C} \cdot t_{\exp }+\mathcal{R}^{2 \mathrm{D}} \cdot \sqrt{\mathbf{D C} \cdot t_{\exp }}$.

To mimic the readout noise, we added the realization of random noise $\mathbf{r n}=\mathcal{R}^{2 \mathrm{D}} \cdot \sigma_{\mathrm{RN}}$ to $\mathbf{I}$ (already here it is not noise-free) in Eq. (17), and it becomes $\mathbf{I}+\mathbf{r n}$.

The quantization effect, or digitization noise, occurs due to quantization of the signal during the $\mathrm{ADC}$ conversion. It is frequently modeled as a random term with $\sigma_{\mathrm{Q}}=\frac{1}{\sqrt{12}} \mathrm{DN}$, which is a standard deviation of a random variable that is uniformly distributed in the range from -0.5 to $0.5 \mathrm{DN}$. However, in our approach, the effect is implemented during the conversion of the detector output into an integer number of DNs. 
Along with the simulated image, we also calculated the variance of the signal in each pixel,

$\mathbf{V a r}=\left(\mathbf{M}+\mathbf{D C} \cdot t_{\mathrm{exp}}\right) \cdot g^{2}+\mathbf{r n}^{2}+\frac{1}{12}$,

where noise-free versions of photo-electrons and dark current are implied.

The realistic simulated image can also be obtained from the noise-free image $\mathbf{I}_{\mathrm{nf}}$, that is, through the second approach, by adding a realization of random noise with the calculated variance $\mathbf{I}=\mathbf{I}_{\mathrm{nf}}+\mathcal{R}^{2 \mathrm{D}} \cdot \sqrt{\mathbf{V a r}}$. We must admit that in this case, the digitization noise is taken into account twice: during the rounding, and from corresponding term in Var. However, this contribution is relatively small, and both approaches for noise implementation give very similar results.

\section{Results}

\subsection{Complete simulated images}

An example of complete simulated images I, calculated for the WBF passband, the synthetic $\mathrm{K}$ corona, and exposure times $t_{\exp }=0.1,1.0$, and $10.0 \mathrm{~s}$. is given in Fig. 12. The horizontal profiles are given in the bottom panel. The logarithmic color scale covers the dynamic range of all three exposures. Using the eclipse $\mathrm{K}$ corona as an input gave very similar results in terms of minimum and maximum intensities and their radial dependences, and noise effect. Thus they are not shown here.

The behavior of the intensity in the images is well understood. At low heights close to the IO, the coronal signal dominates, and the relative contribution of noise is quite small. Higher in the corona, the relative impact of noise becomes increasingly pronounced. For the images with $t_{\exp }=0.1 \mathrm{~s}$, the noise becomes dominant already at $\sim 2 R_{\odot}$. Taking the typical photon flux at these heights and assessing the corresponding contributions of shot noises, we can conclude that the main contribution is the readout noise of the detector.

The interesting result is that the images with an exposure of even $1.0 \mathrm{~s}$ show almost no saturation. The IO vignettes the innermost and brightest corona and efficiently reduces the dynamic range of the registered scene. Thus the default set of exposure times of $0.1,1.0$, and $10.0 \mathrm{~s}$ may need to be reconsidered as two images may be sufficient to cover the whole field of view without saturation. This is discussed in Sect. 8.2.

The images for the Fe XIV filter calculated with and without the $\mathrm{E}$ corona for $t_{\mathrm{exp}}=10 \mathrm{~s}$ are given in Fig. 13. In the right panel the $t_{\exp }=1 \mathrm{~s} \mathrm{WBF}$ image is given for comparison. Despite the narrow spectral passband $(F W H M \sim 1 \mathrm{~nm})$, a $10.0 \mathrm{~s}$ exposure time provides a signal in the range $10-1000 \mathrm{DN}$. The emission of Fe XIV spectral line enhances the intensity in the corresponding regions by a factor $2 \sim 5$. A longer exposure time would deliver an even stronger signal, but the stability of the telescope pointing might not be sufficient to provide sharp images.

\subsection{Scientific processing of ASPIICS data, effect of noise}

On-ground scientific processing of ASPIICS images that converts the measured signal $\mathbf{I}$ into coronal brightness $\mathbf{B}_{\mathrm{W}}$ will in general resemble Eq. (17) applied in the reverse order. The PSF function will not be deconvolved, and the average photometric sensitivity $A$ will be obtained during on-ground calibration of the instrument without decomposition into optical transmittance, geometrical factors, and detector sensitivity. For the sake of demonstration, we omit the detector nonlinearity $\mathcal{L}()$ and express the processing chain as

$\mathbf{B}_{\mathrm{W}}=\left[\frac{\mathbf{B}_{\mathrm{ph}}}{\mathbf{F} \cdot A \cdot t_{\mathrm{exp}}}-\mathbf{B}_{\text {Diff }}-\mathbf{B}_{\mathrm{G}}-\mathbf{B}_{\mathrm{Sc}}\right] \frac{1}{\mathbf{V}}$,

where $\mathbf{B}_{\mathrm{ph}}=\mathbf{I}-\mathbf{D C} \cdot g \cdot t_{\mathrm{exp}}-$ bias is the photo-related part of the registered signal, and the photometric sensitivity factor $A$ also includes the $\mathrm{MSB}_{\mathrm{W}}$ (or the appropriate term for the NBF filter). The term $\mathbf{B}_{\text {tot }}$ in Eq. (17) coincides with $\mathbf{B}_{\mathrm{ph}}$ up to the photometric calibration factor and random noise. We intentionally use different terms to stress the difference between the actually registered optical signal $\mathbf{B}_{\mathrm{ph}}$, which contains random noise, and the physical model $\mathbf{B}_{\text {tot }}$, which in addition might be not fully representative.

In order to understand accuracy of the coronal scene $\mathbf{B}_{\mathrm{W}}$ calculated from the registered or simulated image $\mathbf{I}$, we need to differentiate between noises and other unknown factors. We assume that we observe a coronal scene and register a sequence of several images $\mathbf{I}_{1} . . \mathbf{I}_{N}$ at a significantly high cadence, such that the coronal scene and other parameters do not change during the observations. The actual signal in a given pixel will be slightly different in different images due to the mutual effect of noises. Ideally, the statistical properties of noise in every given pixel should be analyzed from the experimental data; but before any observational data are available, we assume that they are Gaussian distributed and use Var from Eq. (18) for their variance.

Additive components related to optics and the detector do not belong to the coronal signal, and they will be removed during on-ground processing. Thus we define the $\mathrm{S} / \mathrm{N}$ as follows:

$S / N=\frac{\mathbf{B}_{\mathrm{ph}}-\left(\mathbf{B}_{\text {Diff }}+\mathbf{B}_{\mathrm{G}}+\mathbf{B}_{\mathrm{Sc}}\right) \cdot \mathbf{F} \cdot A \cdot t_{\mathrm{exp}}}{\sqrt{\operatorname{Var}}}$.

We created simulated images for the exposure times $t_{\exp }=$ $0.3 \mathrm{~s}$ and $3.0 \mathrm{~s}$ and analyzed their $\mathrm{S} / \mathrm{N}$ and saturation. The results are given in Figs. 14 and 15: The images processed using Eq. (19) are given in the left panels, and the $\mathrm{S} / \mathrm{N}$ in the right panels (note different color scale in the $\mathrm{S} / \mathrm{N}$ panels). The saturated region is small, and the signal there can be restored from the $0.3 \mathrm{~s}$ exposure image, where the $\mathrm{S} / \mathrm{N}$ is also high. Thus the two images can be combined into a full-dynamic-range image that preserves the high $\mathrm{S} / \mathrm{N}$ over the full FOV.

The analysis shows that from a set of two properly exposed images, a high-dynamic-range image with $S / N>20$ can be assembled. This means a relatively small $(<5 \%)$ contribution of noise in every pixel.

\subsection{Effect of the uncertainty in the calibration factors}

Low random noise does not necessarily mean that the accuracy of the final data is improved. We may strongly diminish the noise by averaging a number of registered images $\mathbf{I}_{1}$... $\mathbf{I}_{N}$ or taking an image with sufficiently high $\mathrm{S} / \mathrm{N}$. During the on-ground scientific processing we will use the algorithms following Eq. (19), but the individual parameters in it will be known only to some precision. Their accuracy is determined by a particular method of measurement and corresponding laboratory setup. In addition, some terms of the equation might be not fully correct. For example, due to a different temperature or readout regime, the assumed dark current DC $\cdot g$ might be not correct for a given image. All these uncertainties will appear in every processed image, and their values will be the same from image to image in every given pixel, which means that they provide systematic errors. 


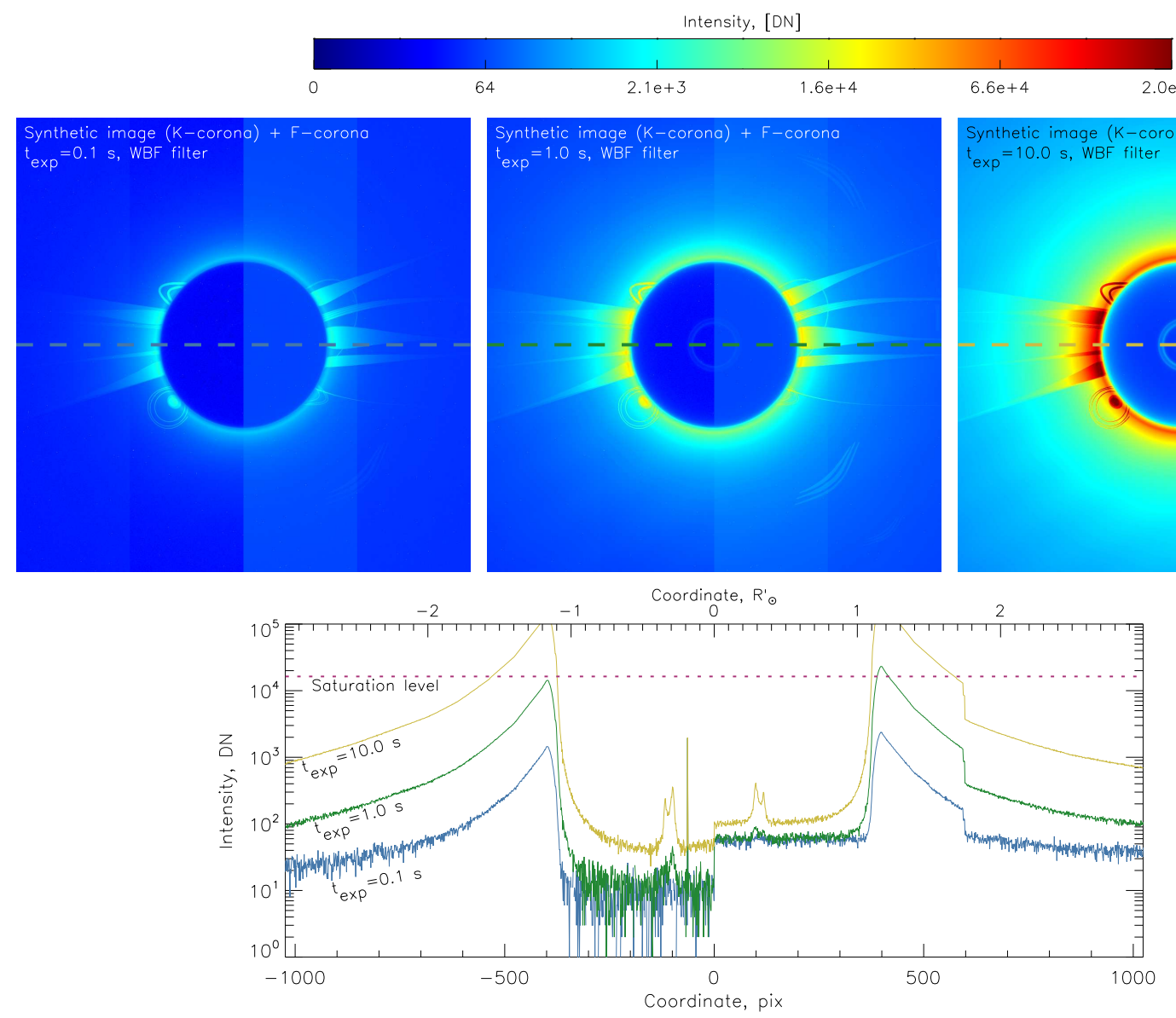

Fig. 12. Examples of ASPIICS simulated data, generated with the described algorithm. The three panels show simulated data generated with a synthetic $\mathrm{K}$ and $\mathrm{F}$ corona and all the other known effects, for the WBF filter. The exposure times are $0.1 \mathrm{~s}($ left), $1.0 \mathrm{~s}$ (middle), and $10.0 \mathrm{~s}$ (right). The bottom panel shows horizontal profiles taken through the center of the images. The color of the lines corresponds to the dashed lines in the images. The saturation level is given, but the signal in the simulated images is not clipped.

The effect of the error of every calibration factor can be evaluated using the formulas for indirect measurements applied to Eq. (19). We consider a particular pixel in the processed image and assume the models of the diffracted light, the ghost light, and the scattered light have absolute errors $\Delta_{\text {Diff }}, \Delta_{\mathrm{G}}$, and $\Delta_{\mathrm{Sc}}$ with respect to their true values. Then the absolute error of the coronal signal $\Delta_{\mathrm{W}}$ is calculated as

$\Delta_{\mathrm{W}}=\Delta_{2}+\Delta_{\text {Diff }}+\Delta_{\mathrm{G}}+\Delta_{\mathrm{Sc}}$

where $\Delta_{2}$ is the absolute error of the term $\frac{B_{\mathrm{ph}}}{F \cdot A \cdot t_{\mathrm{exp}}}$. The errors are summed linearly, in contrast with random noise, for which variance is considered. The error $\Delta_{2}$ is calculated from the relative errors of the radiometric sensitivity $\delta_{\mathrm{A}}$, the flat field $\delta_{\mathrm{F}}$, and the error in the photo-related part of the signal $\delta_{\mathrm{ph}}$,

$\Delta_{2}=\left(\delta_{\mathrm{A}}+\delta_{\mathrm{F}}+\delta_{\mathrm{ph}}\right) \frac{B_{\mathrm{ph}}}{F \cdot A \cdot t_{\mathrm{exp}}}$,

where

$\delta_{\mathrm{ph}}=\left(\Delta_{\mathrm{I}}+\Delta_{\mathrm{DC}}+\Delta_{\text {bias }}\right) / B_{\mathrm{ph}}$.

Here the $\Delta_{I}[D N]$ is the error of the measured signal in the given pixel. The signal is measured directly and thus can only be affected by the random noise. $\Delta_{\mathrm{DC}}$ and $\Delta_{\text {bias }}$ denote the deviation of the assumed dark current and bias from their true values. The last formula is quite important: in regions where the optical signal is weak (both $B_{\mathrm{ph}}$ and $\Delta_{\mathrm{I}}$ are small), possible uncertainties in the dark and bias $\Delta_{\mathrm{DC}}+\Delta_{\text {bias }}$ can affect $\delta_{\text {ph }}$ to a higher degree than the random noise $\Delta_{\mathrm{I}}$. If precise measurements of the coronal signal are desirable, the accuracy of the assumed dark current and bias are more important then the contribution of noise because noise can be removed by averaging. In regions with strong optical signal, the same uncertainty of the dark and bias contributes less to $\delta_{\text {ph }}$ because $B_{\mathrm{ph}}$ is higher.

In Eq. (22) the contribution of the flat-field error will be small in practice because the flat field can be measured almost directly in flight. In contrast, the contribution of the radiometric sensitivity error might be essential. Dedicated calibrations of other coronagraphs were performed on the ground, for example, in the case of LASCO C2 and LASCO C3 (Brueckner et al. 1995), and COR1 (Howard et al. 2008) on board the Solar Terrestrial Relations Observatory (STEREO) satellites. Later on, various methods were applied to perform their in-flight calibration. For example, for the LASCO C2 coronagraph, the F corona and stars were used (Llebaria et al. 2006; Gardès et al. 2013; Colaninno \& Howard 2015), for the LASCO C3, stars were used (Thernisien et al. 2006a), and Jupiter and cross-calibration between STEREO-A and STEREO-B data were used for COR1 (Thompson \& Reginald 2008). The discrepancy between various methods amounted to $10 \%$, thus here we take this value as an estimate for the radiometric calibration accuracy. It is important to stress that this discrepancy is not linked to the repeatability 


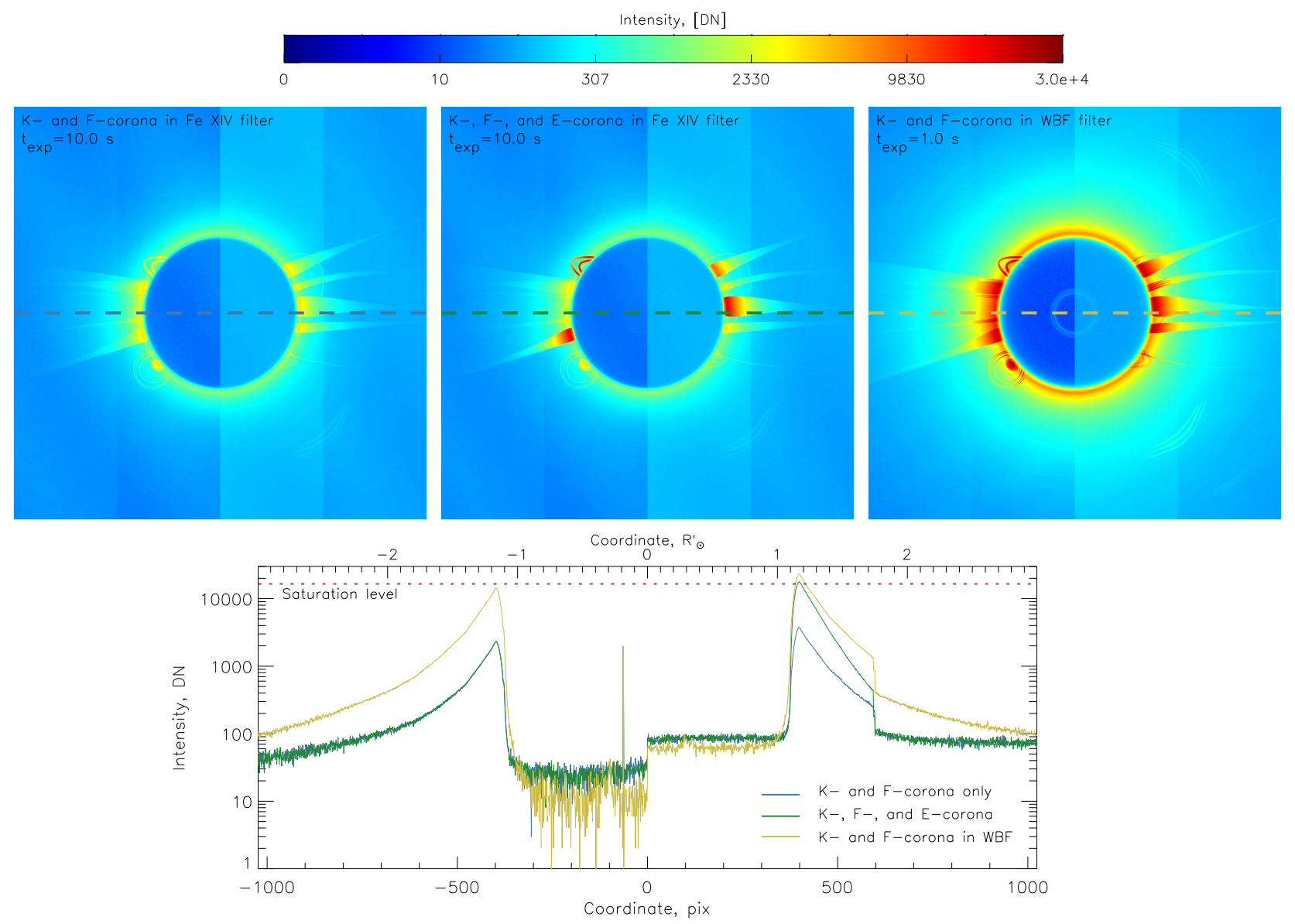

Fig. 13. ASPIICS simulated data, generated for the Fe XIV filter and $t_{\mathrm{exp}}=10.0 \mathrm{~s}$. Left panel: $\mathrm{K}$ (synthetic) and F corona alone were used as input. Middle: K, F, and E corona were used as input. Right: WBF image calculated for the $\mathrm{K}$ and $\mathrm{F}$ corona and $t_{\exp }=1.0 \mathrm{~s}$ (given for reference). Bottom panel: horizontal profiles taken through the center of the images. The color of the lines corresponds to the dashed lines in the images. The logarithmic color scale differs from that in Fig. 12.

of the same measurement, but the discrepancy between the results that were obtained with completely different methods, such as laboratory measurements, calibration using stars, and cross-comparison with other coronagraphs or eclipse observations (Frazin et al. 2012), where the unknown and thus uncontrolled factors are different a priori.

Finally, we considered the effect of other light contributors such as diffraction, ghost light, and scattering. According to Fig. 9, the contribution of scattered light and ghost is small. Hence, if their models have an accuracy on the order of one magnitude (see the discussion in Sect. 9), the errors in their absolute values will not play a large role in the accuracy of the final data. The error in the diffraction model can contribute more, especially closer to the minimal FOV and in the vignetting zone, where the diffraction reaches the level of coronal signal. We discuss possible errors in the diffraction model in Sect. 9 in detail, and here mention a factor 2 as an estimate.

\subsection{Visibility of stars}

In order to analyze the detectability of stars in ASPIICS images, we created an artificial star field with stars situated along the main horizontal, vertical, and diagonals of the FOV, see Fig. 16. This was necessary because there are only a few stars in the realistic images. The artificial star field was propagated through the pipeline. In the artificial star scene, the images of the stars were deliberately positioned outsize the pixel centers, and the
PSF of the telescope was taken into account. The intensity profiles are shown in the right panels in Fig. 16, with the zoomed views in the background. The stars with magnitude $V=4$ can be distinguished in the images starting from heights $\sim 1600$ arcsec $\left(\sim 1.67 R_{\odot}\right)$. At lower heights, the coronal signal and the contribution of noise prevents their detection. Stars with magnitude $V=6$ and dimmer cannot be easily distinguished in the profiles. Their signal might be revealed with a more precise analysis. Furthermore, the realistic flat field and dark current, which vary from pixel to pixel, may also complicate the detection of the stars. The situation improves with increasing exposure time.

\section{Discussion}

Stray light, and in particular, diffracted light, plays a crucial role in the performance of white-light coronagraphs (Bout et al. 2000; Koutchmy \& Belmahdi 1987; Landini et al. 2017b). To improve the accuracy of the stray light removal in other coronagraphs, empirical models in which the minimum backgrounds are derived over several days or weeks are often employed. In ASPIICS, the diffracted light contributes most, and because it depends on the in-orbit configuration of the two spacecraft, a similar strategy cannot be used.

The strongest simplification of the adopted diffraction model (SZ18, as well as its predecessors) is the assumption of an infinitely thin EO. In practice, the EO of ASPIICS has a toroidal 


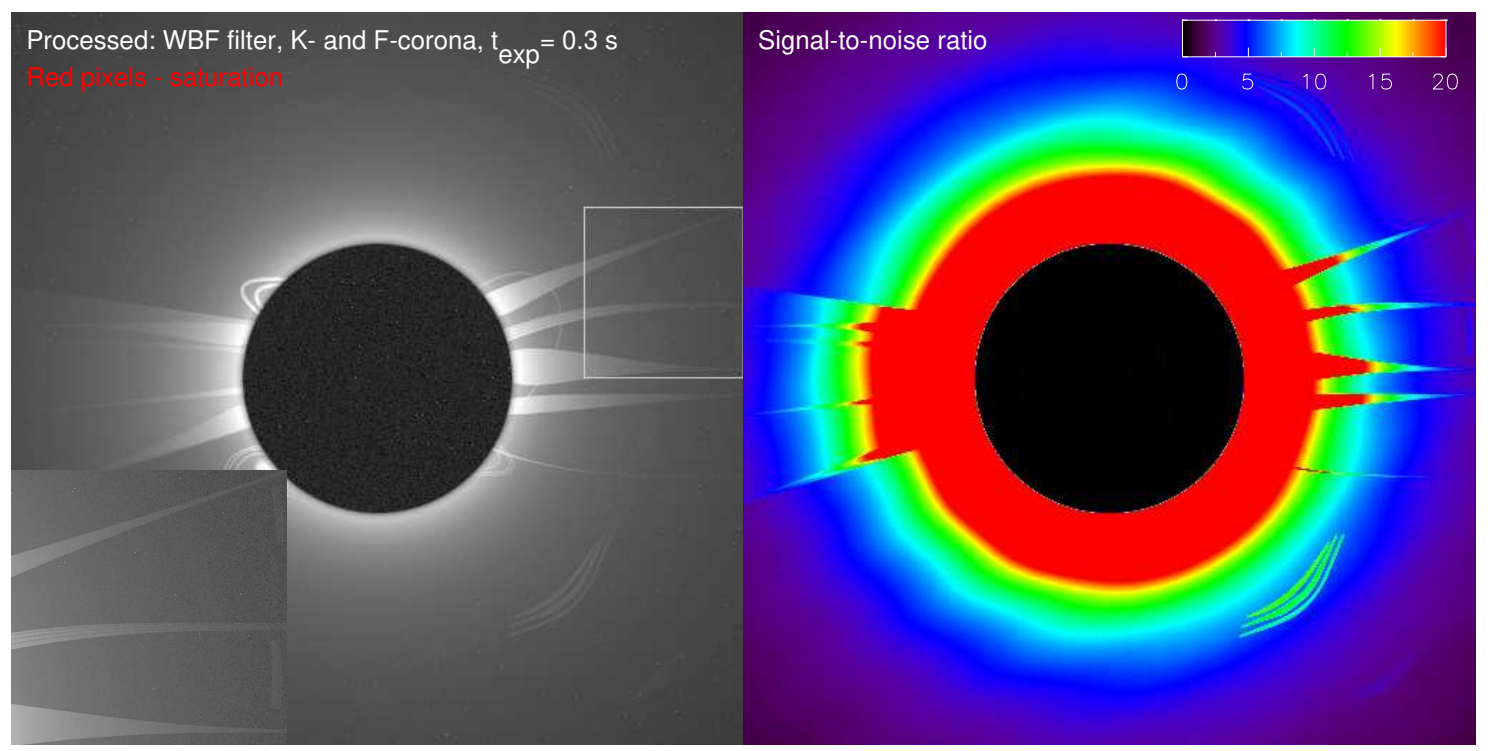

Fig. 14. Processed image (left panel) and signal-to-noise ratio (right panel) for the simulated image (synthetic K-corona, WBF filter) for the $0.3 \mathrm{~s}$ exposure time. In the lower-left corners the zoomed view is given.

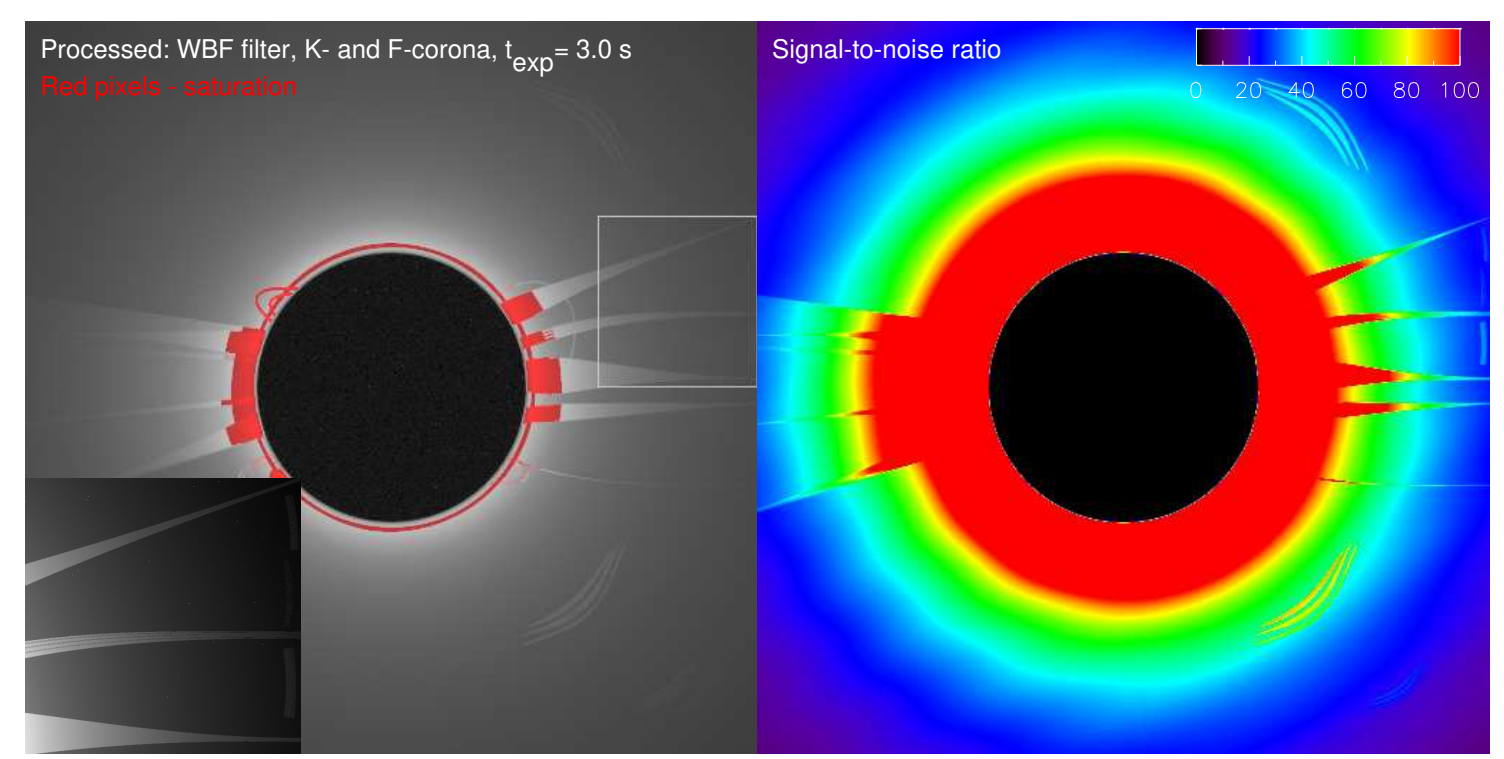

Fig. 15. Same as in Fig. 14, but for $3.0 \mathrm{~s}$ exposure time. In the left panel, the red filling denotes saturated signal. The color table of the S/N has different values with respect to Fig. 14.

shape with $r_{t}=700 \mathrm{~mm}$ (see Galano et al. 2018). The toroidal shape was chosen in order to reduce the amount of the diffracted light that enters the PO, and to reduce the effect of possible small tilts of the EO on the diffraction pattern. Several experimental and analytical results have demonstrated a higher efficiency of thick occulters. In particular, Bout et al. (2000) studied the difference between a single thin disk, several disks, a polished cone, a threaded cone, and a serrated occulter. All the thick occulters were more efficient than the single thin disk. These experiments were performed with real-size occulters and a table-size breadboard. Real-size tests like this are almost impossible for the case of ASPIICS because it is so large. Thus Landini \& Mazzoli (2010) and Landini et al. (2011) compared the efficiency of linear segments of various occulters, and thick occulters were again found to be more efficient, with the advantage being about factor of two. Later, Landini et al. (2016, 2017a) developed an analytical model and showed how an ASPIICS-size configuration can be rescaled to a laboratory size (linear scaling for distances, and quadratic scaling for thicknesses) so that the experiments would be representative. However, in this case, the intensity of the diffracted light can be measured only in the entrance aperture plane and not in the detector plane, as otherwise the remaining optical system would be scaled down to an unrealistic size.

More recent and more advanced analytical and numerical models that consider complex occulters (Rougeot \& Aime (2018) considered a serrated occulter, and Aime (2020) considered several disks) have in general confirmed the experimental results. However, the calculations were also performed in the plane of the entrance aperture, and not in the detector plane.

Unfortunately, it is not fully clear how the diffracted light from the toroidal occulter will behave further in the instrument. SZ18 proposed that in the IO plane the bright diffracted fringe will behave according to the geometrical optics laws, that is, it will always correspond to the reprojected EO edge. Some 


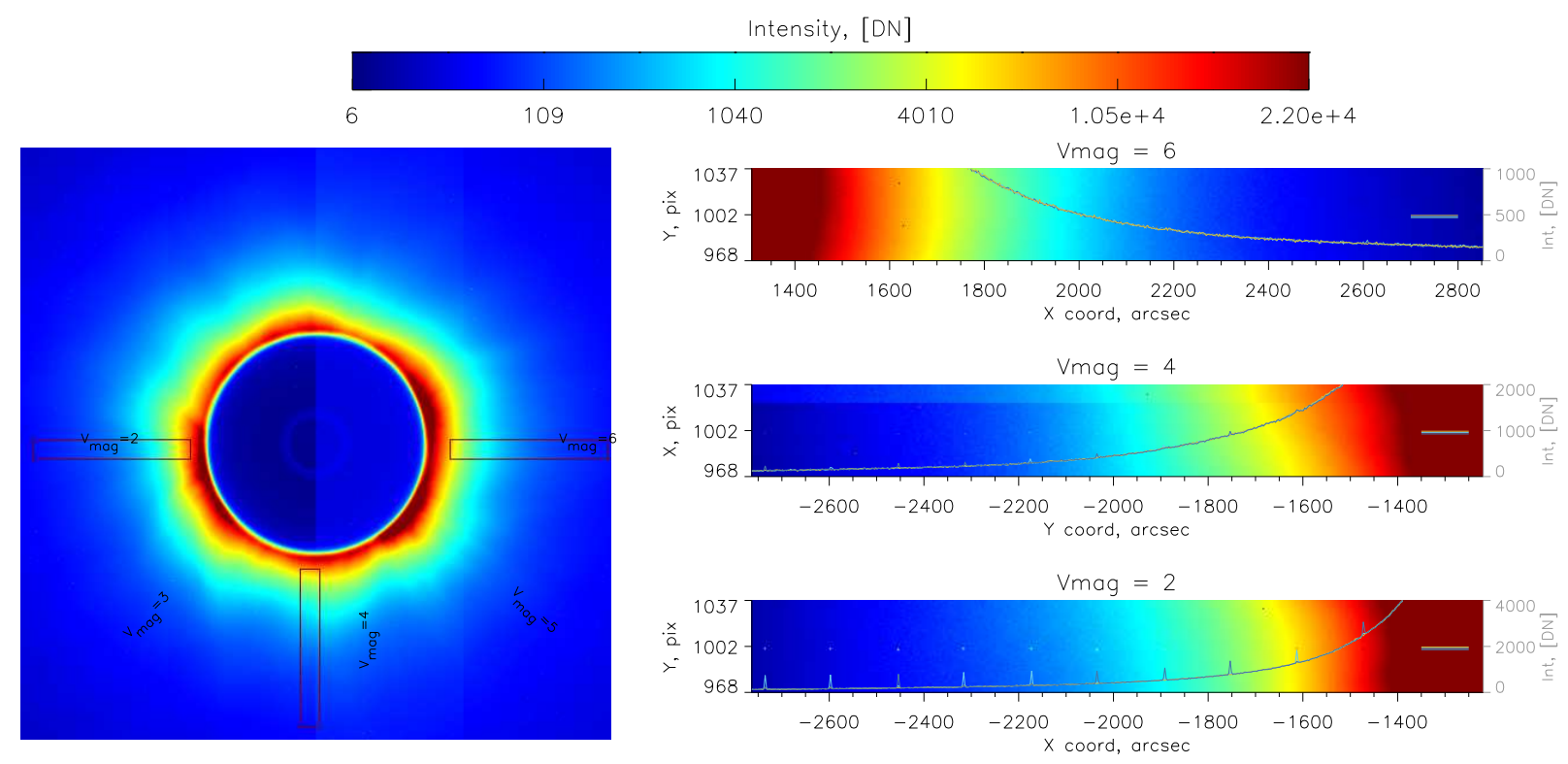

Fig. 16. Analysis of the star visibility in the ASPIICS images in presence of other sources of light and noises. Left panel: complete simulated image (with the eclipse K corona). Right panels: zoomed regions with stars of magnitude $V=6$ (top row), $V=4$ (middle row), and $V=2$ (bottom). The positions of the zoomed regions with respect to the full image are denoted in the left panel. In the zoomed regions, the main axis denotes coordinates, while the right $y$-axis denotes the intensity of the profiles, which is shown with a thin line in each zoomed region. The vertical position of the profiles is denoted by corresponding colored lines in the right part of each zoomed region.

experiments have been foreseen to validate this geometrical behavior, but it is difficult to verify whether the fringe will remain narrow or if the profiles of its wings will change. During further propagation through the instrument, the IO and the Lyot stop also modify the diffracted light. These diaphragms can probably be considered as thin, thus making the results of SZ18 and RR17 applicable (the conclusions of Landini et al. 2017b, who made the optimization of occulters for Metis, support this hypothesis).

The next important contributor is the ghost light. The analytical model of ghost light presented in SH19 assumes a perfectly coaligned optical system and the absence of reflecting mechanical elements. During the adjustments of the Metis coronagraph, Landini et al. (2019) found many reflections from various optical and mechanical components (see their Figs. 7, 11, and 13). They adjusted the position of the IO in the optical layout, which led to a significant improvement of the ghosts. No activities/adjustments to optimize such reflections are foreseen in ASPIICS. However, the foreseen on-ground laboratory tests of the stray light will provide us additional information to improve the model.

In the simulated data pipeline we take possible tilts of the telescope partially into account, that is, only in the calculation of the diffracted light. These tilts will also shift the coronal scene in the images. The effect will not be large, $\sim 10$ pixels for 30 arcsec telescope tilt, therefore we neglected it in the simulated data. During the scientific processing, the coronal images will be recentered based on the data from the navigation systems of the satellite. The telescope tilt does not displace the vignetted zone and the geometrical position of the diffracted fringe on the detector, however.

In our simulated data the flat field includes only the variation of the detector quantum efficiency across the FOV. In general, this effect can include variation of the detector electronic gain $g$ and optical vignetting due to the external occulter or supporting pilons as in the case of LASCO C2 (Llebaria et al. 2004), shading by the filter-supporting mesh as in the Sun Watcher using the Active Pixel System detector and Image Processing (SWAP) telescope (Seaton et al. 2013), and transparency of the filters deposited on the detector as in the TESIS imager (Kuzin et al. 2011). In the case of ASPIICS, the separation of the vignetting from the flat field is required to correct for the diffracted light, which unlike the coronal light, does not experience vignetting. The variation in optical transmittance across the FOV can be included when the corresponding experimental data are available. The assumed simplification of constant gain $g$ throughout the FOV may have a twofold effect. First, it will result in variation of the detector sensitivity over the FOV, which is taken into account by the flat field. Second, the model for noise calculated by Eq. (18) has a scalar gain, but its variation over the FOV should be used. Nevertheless, even under our simplification, the error in the predicted variance will be small because the relative variation of the gain is expected to be up to some dozen percent.

We also aim to determine the ASPIICS characteristics in flight. The front door of ASPIICS is equipped with a highdensity diffuser (HDD), which will allow registering flat-field images under direct Sun illumination (with a closed front door and a special maneuver of the satellites). This will enable deriving the detector flat field and total telescope sensitivity. There are additional possibilities related to the analysis of polarized data. Stray light is considered to be unpolarized, and thus it disappears in the $p B$ and $B$ derived from the polarized data. Comparison of $B$ measured in WBF and polarized images will be used to model the stray light better. A very detailed analysis of LASCO C2 characteristics was recently presented by Lamy et al. (2020). The authors analyzed polarized data registered during 24 years, and derived a refined total sensitivity and even flat field in individual polarized channels of LASCO C2 (LASCO C2 is equipped with three polarizers at $60^{\circ}$ ). We hope that a similar analysis will be possible using ASPIICS data.

The model of the detector is currently limited as well: the flat field, dark current, and bias are represented by smooth 2D arrays and do not include the dependence on temperature or other settings. However, the temperature dependence is relatively simple to implement both in the simulated data pipeline 
and in scientific processing when measurements are available. Our analysis shows that these effects are weak in the overall signal. It is worth mentioning that ASPIICS will be thermally stabilized and the detector will be passively cooled below $-35^{\circ} \mathrm{C}$ during operations. Thus the dark current and its variations will be minimized. We do not consider optical effects that are unknown to us, for example, additional stray light formed by the reflections from the mechanical surfaces or blinking edges.

The analysis of the effect of the uncertainties of the calibration parameters and the noise shows that for the WBF passband, the greatest uncertainty for properly exposed images will be determined by the accuracy of the radiometric sensitivity. For the NBF passbands, where the photon flux is lower, random noise will also start to play a role. Making an exposure even longer (than the foreseen $10 \mathrm{~s}$ ) might be not possible because of the stability of the satellite. We note that these accuracies concern the primary product, that is, the brightness of the corona in the whole passband. Higher-level products, such as the separated $\mathrm{K}$ corona, or polarization parameters may have a different level of noise, and will also depend on the validity of the assumed models. For example, Inhester et al. (2021) recently showed that degree of polarization $p$, derived from three images with different polarization, is biased toward higher values in the regions of low $p$ by the specific propagation of random errors to the polarized products.

\section{Conclusions}

We presented the approach to generating simulated data of the PROBA-3/ASPIICS coronagraph. The simulated data were produced with realistic assumptions and available physical models. The simulation pipeline used realistic coronal images as input, took optical effects such as diffracted light, ghost, and scattered light into account, and also characteristics such as optical throughput, PSF, vignetting, and the characteristics of the detector such as sensitivity, dark current, and bias. The noise was introduced based on physical mechanisms, such as Poisson shot noise of photons and Gaussian readout noise.

The analysis of the simulated images allowed us to compare the effect of different stray-light components, and to evaluate the uncertainty of the final data (i.e., after the on-ground processing) due to random noise and systematic errors. The calculated S/N maps show that during nominal synoptic observations, two WBF images with $t_{\exp }=0.3 \mathrm{~s}$ and $3.0 \mathrm{~s}$ will be sufficient to assemble a full FOV high-dynamic-range image with $S / N>20$ across the FOV. The error in the final data due to the uncertainty of the calibration factors, such as photometric sensitivity, inappropriate bias, or dark current maps, may be also essential and may limit the accuracy of the final data. At the same time, the expected level of stray light is not expected to play a significant role. The main component of the stray light will be due to diffraction, which has the strongest intensity in the inner FOV zone, where the coronal signal is high.

The expected performances of ASPIICS will be verified during the in-flight commissioning. The synthetic data pipeline will be fed with real calibration data upon their availability.

Acknowledgements. The authors thank the European Space Agency (ESA) and the Belgian Federal Science Policy Office (BELSPO) for their support in the framework of the PRODEX Programme. We are grateful to our ESA colleagues D. Galano, J. Versluys, R. Rougeot, and C. Thizy and C. Galy from CSL for their support during preparation of our models and calibration activities, and to Dr. B. Decraemer for providing the data for polarized images. PROBA-3 is developed by a large consortium of 36 companies with a core team of five companies that includes SENER as Prime with QinetiQ Space nv, Airbus Defence and Space Spain, GMV Space and Defence and Spacebel. The payload industrial consortium is led by Centre Spatial de Liège.

\section{References}

Aime, C. 2013, A\&A, 558, A138

Aime, C. 2020, A\&A, 637, A16

Aime, C., Theys, C., Rougeot, R., \& Lantéri, H. 2019, A\&A, 622, A212

Allen, C. W. 1976, Astrophysical Quantities, 3rd edn. (Athlon)

Antonucci, E., Romoli, M., Andretta, V., et al. 2020, A\&A, 642, A10

Bayanna, A. R., Mathew, S. K., Sankarasubramanian, K., et al. 2011, Exp. Astron., 29, 145

Billings, D. E. 1966, A guide to the solar corona

Bout, M., Lamy, P., Maucherat, A., Colin, C., \& Llebaria, A. 2000, Appl. Opt., 39,3955

Brueckner, G. E., Howard, R. A., Koomen, M. J., \& Korendyke, C. M. 1995, Sol. Phys., 162, 357

Bryson, S. T., Jenkins, J. M., Peters, D. J., et al. 2010, in Modeling, Systems Engineering, and Project Management for Astronomy IV, eds. G. Z. Angeli, P. Dierickx, et al., 7738, 773808

Colaninno, R. C., \& Howard, R. A. 2015, Sol. Phys., 290, 997

Cox, A. N. 2000, Allen's Astrophysical Quantities (New York: AIP Press; Springer)

Decraemer, B., Zhukov, A. N., \& Doorsselaere, T. V. 2019, ApJ, 883, 152

ESA, 1997, in The HIPPARCOS and TYCHO catalogues. Astrometric and photometric star catalogues derived from the ESA HIPPARCOS Space Astrometry Mission, ESA Spec. Pub., 1200

Frazin, R. A., Vásquez, A. M., Thompson, W. T., et al. 2012, Sol. Phys., 280, 273

Futyan, D., Fortier, A., Beck, M., et al. 2020, A\&A, 635, A23

Galano, D., Buckley, S., Cernica, I., et al. 2018, in Space Telescopes and Instrumentation 2018: Optical, Infrared, and Millimeter Wave, eds. H. A. MacEwen, M. Lystrup, G. G. Fazio, et al. (SPIE), Proc. SPIE, 10698, $106982 \mathrm{Y}$

Galano, D., Jollet, D., Mellab, K., et al. 2019, in 10th International Workshop on Satellite Constellations and Formation Flying (IWSCFF)

Galy, C., Fineschi, S., Galano, D., et al. 2015, in Solar Physics and Space Weather Instrumentation VI, Proc. SPIE, 9604, 96040B

Galy, C., Thizy, C., Stockman, Y., et al. 2019, in International Conference on Space Optics \&mdash; ICSO 2018, SPIE Conf. Ser., 11180, 111802H

Gardès, B., Lamy, P., \& Llebaria, A. 2013, Sol. Phys., 283, 667

Golub, L., \& Pasachoff, J. 1997, The Solar Corona (Cambridge University Press) Guhathakurta, M., Holzer, T. E., \& MacQueen, R. M. 1996, ApJ, 458, 817

Howard, R. A., Moses, J. D., Vourlidas, A., et al. 2008, Space Sci. Rev., 136, 67 Inhester, B., Mierla, M., Shestov, S., \& Zhukov, A. N. 2021, Sol. Phys., 296, 72 Jejčič, S., Heinzel, P., Labrosse, N., et al. 2018, Sol. Phys., 293

Jenkins, J. M., Tenenbaum, P., Caldwell, D. A., et al. 2018, Res. Notes AAS, 2, 47

Kim, I. S. 1997, in NATO Advanced Science Institutes (ASI) Series C, eds. Z. Mouradian, \& M. Stavinschi, NATO ASI Ser. C, 494, 159

Koutchmy, S. 1988, Space Sci. Rev., 47, 95

Koutchmy, S., \& Belmahdi, M. 1987, J. Opt., 18, 265

Kuzin, S. V., Shestov, S. V., Bogachev, S. A., et al. 2011, Sol. Syst. Res., 45, 174

Lamy, P., Damé, L., Vivès, S., \& Zhukov, A. 2010, in Space Telescopes and Instrumentation 2010: Optical, Infrared, and Millimeter Wave, Proc. SPIE, 7731,773118

Lamy, P., Llebaria, A., Boclet, B., et al. 2020, Sol. Phys., 295, 89

Landini, F., \& Mazzoli, A. 2010, in Ground-based and Airborne Instrumentation for Astronomy III, Proc. SPIE, 7735, 77354D

Landini, F., Vives, S., Venet, M., et al. 2011, Appl. Opt., 50, 6632

Landini, F., Romoli, M., Baccani, C., et al. 2016, Opt. Lett., 41, 757

Landini, F., Vives, S., Romoli, M., et al. 2017a, in International Conference on Space Optics - ISCO 2012, Proc. SPIE, 10564, 105640F

Landini, F., Baccani, C., Schweitzer, H., et al. 2017b, Opt. Lett., 42, 4800

Landini, F., Romoli, M., Fineschi, S., et al. 2019, in International Conference on Space Optics - ICSO 2018, eds. N. Karafolas, Z. Sodnik, B. Cugny, et al. (SPIE), 39, 89

Llebaria, A., Lamy, P. L., \& Bout, M. V. 2004, in Telescopes and Instrumentation for Solar Astrophysics, eds. S. Fineschi, \& M. A. Gummin, Proc. SPIE, 33, 5171-12

Llebaria, A., Lamy, P., \& Danjard, J. F. 2006, Icarus, 182, 281

Perryman, M. A. C., Lindegren, L., Kovalevsky, J., et al. 1997, A\&A, 500, 501

Pfisterer, R. N. 2011, Opt. Eng., 16

Piqueras, J. J. M. 2013, PhD Thesis, Technischen Universität Carolo-Wilhelmina zu Braunschweig zur Erlangung der Würde, The address of the publisher

Renotte, E., Alia, A., Bemporad, A., et al. 2015, in Solar Physics and Space Weather Instrumentation VI, Proc. SPIE, 9604, 96040A

Rougeot, R., \& Aime, C. 2018, A\&A, 612, A80 
Rougeot, R., Flamary, R., Galano, D., \& Aime, C. 2017, A\&A, 599, A2

Rougeot, R., Galano, D., Kirschner, V., et al. 2018, in Space Telescopes and Instrumentation 2018: Optical, Infrared, and Millimeter Wave, eds. H. A. MacEwen, M. Lystrup, G. G. Fazio, et al., Proc. SPIE, 10698, 106982T

Rougeot, R., Flamary, R., Mary, D., \& Aime, C. 2019, A\&A, 626, A1

Seaton, D. B., Berghmans, D., Nicula, B., et al. 2013, Sol. Phys., 286, 43

Shestov, S. V., \& Zhukov, A. N. 2018, A\&A, 612, A82

Shestov, S. V., Zhukov, A. N., \& Seaton, D. B. 2019, A\&A, 622, A101

Shestov, S., Bourgoinie, B., Nicula, B., et al. 2020, in Space Telescopes and Instrumentation 2020: Optical, Infrared, and Millimeter Wave, eds. M. Lystrup, N. Batalha, E. C. Tong, N. Siegler, M. D. Perrin, et al. (SPIE), 4, 264 Solanki, S. K., del Toro Iniesta, J. C., Woch, J., et al. 2020, A\&A, 642, A11

Thernisien, A. F., Patel, N. S., Howard, R. A., Marqué, C., \& Vourlidas, A. 2004, AGU Fall Meeting Abstracts, 2004, SH21B-0404

Thernisien, A. F. R., Howard, R. A., \& Vourlidas, A. 2006a, ApJ, 652, 763

Thernisien, A. F., Morrill, J. S., Howard, R. A., \& Wang, D. 2006b, Sol. Phys. 233,155

Thompson, W. T. 2018, Sol. Phys., 293, 1

Thompson, W. T., \& Reginald, N. L. 2008, Sol. Phys., 250, 443

Tiraplegui, S., Serrano, D., Peñin, L. F., et al. 2019, in 8th European Conference for Aeronautics and Aerospace Sciences (EUCASS)

\section{Appendix A: Spectral sensitivity of ASPIICS and the coronal brightness in a passband}

In order to derive the photometric sensitivity of ASPIICS for a given passband, we made the following assumptions: The brightness of the coronal scene is given by $B_{\mathrm{Cor}}(\lambda)$, and the optical system of the telescope has a spectral optical transmittance $T(\lambda)$ and quantum efficiency $\mathrm{QE}(\lambda)$. Characteristics such as aperture size, pixel size, detector gain, and dark current do not depend on wavelength. Then the measured signal (its photo-related part) can be expressed as

$I=\left[\int B_{\mathrm{Cor}}(\lambda) T(\lambda) \mathrm{QE}(\lambda) \mathrm{d} \lambda\right] \cdot \mathrm{d} S_{\text {pix }} \cdot A p \cdot g \cdot t_{\text {exp }}$.

Because the passbands of ASPIICS are relatively narrow, we can convert the integral over $\mathrm{d} \lambda$ into coronal brightness in the given passband $B_{\mathrm{Cor}}^{\mathrm{f}}$ and the sensitivity $A_{\mathrm{f}}$,

$\begin{aligned} I & =\left[\int_{\lambda_{1}}^{\lambda_{2}} B_{\mathrm{Cor}}(\lambda) T^{\prime}(\lambda) \mathrm{d} \lambda\right] \cdot T_{\mathrm{f}} \cdot \mathrm{d} S_{\text {pix }} \cdot A p \cdot \mathrm{QE}_{\mathrm{f}} \cdot g \cdot t_{\text {exp }} \\ & =B_{\text {Cor }}^{\mathrm{f}} \cdot A_{\mathrm{f}} \cdot t_{\text {exp }}\end{aligned}$

where the coronal brightness for a given passband is defined as $B_{\text {Cor }}^{\mathrm{f}}=\int_{\lambda_{1}}^{\lambda_{2}} B_{\mathrm{Cor}}(\lambda) T^{\prime}(\lambda) \mathrm{d} \lambda$, and $T^{\prime}(\lambda)$ is the renormalized spectral transmittance of the optical system, such that $\max \left(T^{\prime}\right)=1$, $T_{\mathrm{f}}$ is the maximum transmittance, and finally, the radiometric sensitivity of the telescope is $A_{\mathrm{f}}=T_{\mathrm{f}} \cdot \mathrm{d} S_{\text {pix }} \cdot A p \cdot \mathrm{QE}_{\mathrm{f}} \cdot g$ in the given passband. 